\title{
Sensitivity of Single Coil Electromagnetic Sensors for Breathing Measurements
}

by

\author{
Rosalyn Margaret Seeton, BASc, University of British Columbia
}

\author{
A thesis submitted to the \\ Faculty of Graduate Studies and Research \\ in partial fulfillment of the requirements for the degree of \\ Master of Applied Science in Biomedical Engineering \\ Ottawa-Carleton Institute for Biomedical Engineering \\ Department of Systems and Computer Engineering \\ Carleton University \\ Ottawa, Ontario, Canada \\ December 2008
}

(C) Copyright 2008, Rosalyn M. Seeton 


$\begin{array}{ll}\begin{array}{l}\text { Library and } \\ \text { Archives Canada }\end{array} & \begin{array}{l}\text { Bibliothèque et } \\ \text { Archives Canada }\end{array} \\ \begin{array}{l}\text { Published Heritage } \\ \text { Branch }\end{array} & \begin{array}{l}\text { Direction du } \\ \text { Patrimoine de l'édition }\end{array} \\ \begin{array}{l}\text { 395 Wellington Street } \\ \text { Ottawa ON K1A 0N4 } \\ \text { Canada }\end{array} & \begin{array}{l}\text { 395, rue Wellington } \\ \text { Ottawa ON K1A 0N4 } \\ \text { Canada }\end{array}\end{array}$

Your file Votre référence ISBN: 978-0-494-47521-8 Our file Notre référence ISBN: 978-0-494-47521-8

NOTICE:

The author has granted a nonexclusive license allowing Library and Archives Canada to reproduce, publish, archive, preserve, conserve, communicate to the public by telecommunication or on the Internet, loan, distribute and sell theses worldwide, for commercial or noncommercial purposes, in microform, paper, electronic and/or any other formats.

The author retains copyright ownership and moral rights in this thesis. Neither the thesis nor substantial extracts from it may be printed or otherwise reproduced without the author's permission.
AVIS:

L'auteur a accordé une licence non exclusive permettant à la Bibliothèque et Archives Canada de reproduire, publier, archiver, sauvegarder, conserver, transmettre au public par télécommunication ou par l'Internet, prêter, distribuer et vendre des thèses partout dans le monde, à des fins commerciales ou autres, sur support microforme, papier, électronique et/ou autres formats.

L'auteur conserve la propriété du droit d'auteur et des droits moraux qui protège cette thèse. $\mathrm{Ni}$ la thèse ni des extraits substantiels de celle-ci ne doivent être imprimés ou autrement reproduits sans son autorisation.
In compliance with the Canadian Privacy Act some supporting forms may have been removed from this thesis.

While these forms may be included in the document page count, their removal does not represent any loss of content from the thesis.
Conformément à la loi canadienne sur la protection de la vie privée, quelques formulaires secondaires ont été enlevés de cette thèse.

Bien que ces formulaires aient inclus dans la pagination, il n'y aura aucun contenu manquant.

\section{Canada}


The undersigned recommend to the

Faculty of Graduate Studies and Research

acceptance of the thesis

\section{Sensitivity of Single Coil Electromagnetic Sensors for Breathing Measurements}

Submitted by Rosalyn Margaret Seeton, BASc, University of British Columbia in partial fulfillment of the requirements for the degree of

Master of Applied Science in Biomedical Engineering

Thesis Supervisor

Dr. Andy Adler

Chair, Department of Systems and Computer Engineering

Dr. Victor Aitken

2008, Carleton University 


\section{Abstract}

We propose a novel approach to optimizing the sensitivity of a single coil sensor based on a theoretical model derived from electromagnetic theory. The thorax is subject to variations in conductivity due to respiratory and cardiac activities and our electromagnetic sensor is designed to measure these changes. We derive a theoretical model for changes in impedance of a current carrying coil caused by a volume conductor located axially above the coil. We model the effect that changes to coil impedance have on the frequency of oscillating circuits and we design two circuits that will optimize this effect. We test our theoretical model by using conductive phantoms to measure frequency changes in our sensor. Finally, we test our sensor's ability to de-

tect changes in lung volume through in vivo trials with six participants performing breathing manoeuvers. 


\section{Acknowledgements}

I would like to dedicate this thesis to my Nana, who never fails to tell me how clever I am, and to my parents, who have supported me with encouragement, constructive criticism, and the occasional financial boost.

I would like to thank Dr. Andy Adler for all his support (academic, financial, moral and otherwise), and for his "sanity checks". His guidance and practical approach were crucial to the completion of this thesis. I would also like to thank Nagui Mikhail, the Hardware Manager from the Department of Electronics, for giving me access to lab materials and equipment as well as providing me with technical assistance. Finally, I would like to acknowledge Dr. Monique Frize for her encouragement and for funding me on other projects during my graduate studies.

My boyfriend, Laurence Smith, has supported me throughout my graduate studies, from giving technical help when I was frustrated to providing meals when I did not have time to feed myself. Laurie motivated me and kept me on track and healthy. My brother and sister also encouraged me and inspired me with their accomplishments. I would like to thank both my family and Laurie's family for all their support and also for providing much needed rest and relaxation during holidays. 


\section{Contents}

1 Introduction $\quad 1$

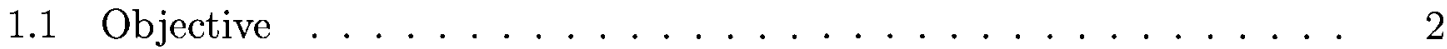

1.2 Summary of Contents . . . . . . . . . . . . . . . 2

2 Background Information 4

2.1 Sleep Apnea . . . . . . . . . . . . . . . . . . . . . 4

2.2 Methods of Respiratory Monitoring . . . . . . . . . . . . . . 5

2.3 Methods of Noncontact Respiratory Monitoring . . . . . . . . . . 6

2.4 Respiratory Monitoring by Magnetic Induction . . . . . . . . . . . . . 7

2.5 Summary ............................. 10

3 Theoretical Model $\quad 12$

3.1 Review of Electrodynamics . . . . . . . . . . . . . . 13

3.2 Theoretical Model . . . . . . . . . . . . . . . . 14

3.3 Implementation of Theoretical Model . . . . . . . . . . . . 20

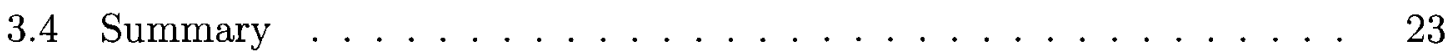

4 Electronic Design $\quad 24$

4.1 Review of Oscillators . . . . . . . . . . . . . 25

4.2 Colpitts Oscillator . . . . . . . . . . . . . 26 
4.2 .1 Frequency . . . . . . . . . . . . . . . . . . 28

4.2 .2 Circuit Optimization . . . . . . . . . . . . . . 32

4.3 Relaxation Oscillator . . . . . . . . . . . . . . . . . . . . 33

4.3 .1 Frequency . . . . . . . . . . . . . . . . 34

4.3 .2 Circuit Optimization . . . . . . . . . . . . . . . 40

4.4 Inductive Coil . . . . . . . . . . . . . . . . . . . 41

4.5 Component Selection . . . . . . . . . . . . . . . 43

4.6 Frequency Measurement . . . . . . . . . . . . . . . 44

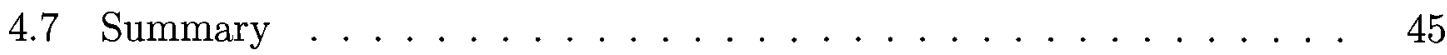

5 Experiments 46

5.1 Phantom Trials . . . . . . . . . . . . . . . . . . . 46

$5.1 .1 \quad$ Experimental Setup . . . . . . . . . . . . . . . . 47

$5.1 .2 \quad$ Phantom Trial $\# 1 \ldots \ldots \ldots \ldots$

$5.1 .3 \quad$ Phantom Trial $\# 2 \ldots \ldots \ldots \ldots \ldots$

5.1 .4 Phantom Trial \#3 $\ldots \ldots \ldots \ldots \ldots$

5.2 In Vivo Trials . . . . . . . . . . . . . . . . . . 58

$5.2 .1 \quad$ Experimental Setup . . . . . . . . . . . . . . 58

5.2 .2 In Vivo Trial Results . . . . . . . . . . . . . . 61

5.2 .3 Statistical Analysis . . . . . . . . . . . . . . . 64

5.2.4 Discussion of In Vivo Trial Results . . . . . . . . . . . 65

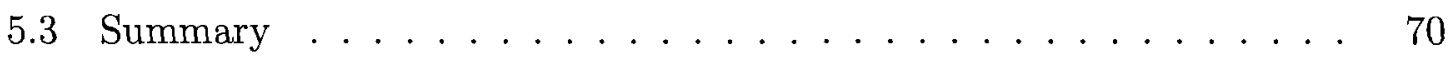

$\begin{array}{lll}6 & \text { Discussion } & 71\end{array}$

6.1 Summary of Contributions . . . . . . . . . . . . . . 71

6.2 Comparison of Model and Experimental Results . . . . . . . . 72

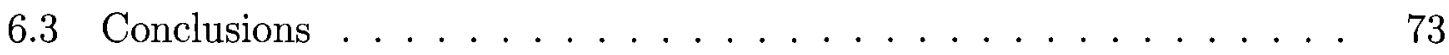


6.4 Future Research . . . . . . . . . . . . . . . 73

A

A.1 Matlab Code . . . . . . . . . . . . . . . 75

B

B.1 Letter of Information and Consent . . . . . . . . . 79

References 


\section{Chapter 1}

\section{Introduction}

Many non-invasive sensors have been developed for respiratory monitoring but the most reliable among these are techniques that suffer from undesirable restrictions to the patient such as bands or electrodes attached to the chest, or face masks and sensors attached near oral cavities. Reliable methods of noncontact respiratory monitoring are needed for applications such as sleep and apnea monitoring. Apnea is the temporary cessation of breathing and sleep apnea is a prevalent condition that has been associated with various detrimental effects.

Electromagnetic sensors provide a noncontact method of measuring changes in conductivity associated with respiratory and cardiac activity but they suffer from low signal strength and are highly subjective to noise. Various electromagnetic sensor designs have been developed for numerous biomedical applications. However, due to the complex nature of the electromagnetic interactions and the unknown geometries of conductive objects, the sensor behaviours are not fully understood in terms of what their signals are actually measuring. The electromagnetic models developed for these sensors have not been exploited to optimize sensor design.

This thesis investigates the sensitivity of a single coil electromagnetic sensor for 
noncontact monitoring of breathing. The coil of the sensor is meant to be placed under the lungs of a participant in the supine position in order to detect changes in conductivity of the thorax and correlate these to lung volume changes. The primary applications of this sensor are for sleep and apnea monitoring.

\subsection{Objective}

The goals of this thesis are to:

- Develop a theoretical model of coil sensitivity to nearby conductivity changes

- Develop a model of oscillator circuit sensitivity from which optimal component values may be selected

- Design and interpret the electrical circuits used as oscillators

- Conduct phantom tests to evaluate the sensitivity of the sensor and validate the theoretical model

- Conduct in vivo tests to measure lung volume changes with the sensor and to compare these to the theoretical model

\subsection{Summary of Contents}

Chapter 2 reviews the background information of relevant research in the field of respiratory monitoring with particular emphasis on non-invasive techniques used for sleep and apnea monitoring. Other electromagnetic sensors are described. Chapter 3 presents our theoretical model. Some basic electromagnetic principles are reviewed and the governing equation of the theoretical model is derived using electromagnetic 
theory. The implementation steps are discussed and some results of the theoretical model are given. Chapter 4 details the electronic designs for our sensor. Several sensor designs were investigated and two designs were chosen. The two circuits are analyzed and an approach to maximize the sensitivity of the sensors is presented. The final component selections are included. Chapter 5 describes all the phantom and in vivo experiments that were conducted with our sensors. The experimental setup, theoretical predictions, measured results, and discussion are provided for all the trials. A statistical analysis of the in vivo results is also included. Chapter 6 discusses our theoretical and experimental results, summarizes our contributions, and provides suggestions for future research. 


\section{Chapter 2}

\section{Background Information}

There are many pulmonary tests needed to examine lung function or screen for or diagnose various lung conditions. Imaging techniques, including various forms of $\mathrm{X}$-Ray and Magnetic Resonance Imaging, are primarily used for visualizing anatomical structures or physiological responses. Other tests, such as Pulmonary Function Tests, body plethysmography, or nitrogen washout, are mainly concerned with measuring lung capabilities like the total lung capacity or the gas transfer. Respiratory monitoring techniques are used for various clinical situations such as monitoring patients during and after sedation, monitoring patients with trauma or severe infections, monitoring premature infants, and monitoring for infant and adult apnea [7]. This chapter will review the current methods of respiratory monitoring, and particularly those aimed at sleep and apnea monitoring.

\subsection{Sleep Apnea}

Sleep apnea is the cessation of breathing or inadequate breathing during sleep. Adult sleep apnea is a widespread condition that decreases sleep quality and it has been 
linked with systemic hypertension, cardiovascular disease, stroke, and abnormal glucose metabolism [23]. Apnea in infants is associated with Sudden Infant Death Syndrome [30]. There is significant interest in monitoring breathing during sleep to detect and measure sleep apnea. Sleep monitoring and apnea sensing requires a method of supervising a patient without interfering with their natural state. In other words, an ideal sensor for these applications would be non-invasive, minimally restrictive, robust enough to compensate for patient movement, and would function without relying on patient cooperation. It is also desirable to make the system simple to set up and as autonomous as possible in terms of data interpretation.

\subsection{Methods of Respiratory Monitoring}

Common non-invasive techniques of respiratory monitoring are respiratory inductance plethysmography, impedance plethysmography, photoplethysmography, and flow sensors such as nasal thermistor. These techniques have been proven to be effective and are often used to compare the performance of new respiratory monitoring devices. Respiratory inductance plethysmography is accomplished by wrapping elastic bands embedded with wires around the the abdomen and the rib cage and measuring the self inductance of the bands to estimate the cross-sectional area of the thorax [4]. Impedance plethysmography involves sending low current, high frequency signals across two or more electrodes on the thorax in order to measure the transthoracic impedance[4]. With photoplethysmography, a fiber-optic sensor, which detects blood volume changes by infrared scattering and absorption properties, is attached to the finger and the respiratory signal is extracted [3]. Airflow sensors, such as pneumotachs, are usually attached near the patient's airway using a mouthpiece or face mask. Nasal thermistors utilize the temperature difference between inspired and expired air, 
while other sensors take advantage of changes in $\mathrm{CO}_{2}$, humidity, or sounds to measure airflow [7].

The main shortcomings of all these techniques is that they demand at least a minimal amount of patient cooperation and they require some form of contact with the patient, which poses undesirable restrictions. Each method has other drawbacks as well. A good review of the advantages and disadvantages of these and other techniques is given by Folke et al [7].

\subsection{Methods of Noncontact Respiratory Monitor- ing}

A promising noncontact respiratory monitoring technique involves the use of pressure sensors in a mattress to measure the respiratory rate, heart rate, and movement [28][15]. These systems typically have one or more sensors arrayed in a mat placed above or below the mattress. They have the advantage of not restricting the patient in any way, but measurements of respiratory and heart rates are affected by patient movement [15].

There are various other non-invasive respiratory monitoring techniques that have been studied include using a ceiling-attached microwave antenna to monitor respiratory signs [32], using a ultrasonic sensor to monitor for sleep apnea [20], using thermal infrared imaging to monitor breathing by detecting $\mathrm{CO}_{2}$ emissions [6], using a charge-coupled device camera to visually monitor respiratory patterns in real-time [21]. 


\subsection{Respiratory Monitoring by Magnetic Induc- tion}

Noncontact sensors based on magnetic induction have also been made for respiratory monitoring. Often referred to as electromagnetic sensors or eddy current sensors, they can provide unobtrusive monitoring of lung activity and are therefore good potential candidates for sleep and apnea monitoring.

Electromagnetic sensors use an inductive coil that carries a time varying current to produce a changing magnetic field. Conductive materials subjected to this dynamic magnetic field experience eddy currents to counteract the changes in flux. This effect can be detected in a receiving coil and the resulting measurement is a function of the conductivity of the material. Compared to conventional biomedical techniques of measuring conductivity, electromagnetic sensors have the advantage of not requiring electrodes and the signal is also unattenuated by bone and skin. On the other hand, since magnetic fields dissipate with the square of distance, electromagnetic sensors are limited in their range.

The more well-known uses of electromagnetic sensors that rely on eddy currents are for positioning or proximity measurement, and for non-destructive testing of conductive materials [25]. These applications involve very conductive targets and the sensors can be optimized to provide very precise measurements.

The activities of the heart and lungs cause small conductivity changes within the body that can be detected by an electromagnetic sensor. One of the early biomedical magnetic induction sensors, developed by Tarjan and McFee was for measuring conductivity of the head and torso [31]. It used a crystal oscillator to drive an excitation coil and receiving coils, mounted coaxially to the excitation coil, measured the induced signal like a gradiometer. The resistivity of the torso was measured by 
assuming homogeneous conductivity and calibrating the sensor with saline phantoms. They tested the sensor's capabilities with in vivo measurements of the head resistivity and changes in torso conductivity. The sensor measured minimal changes in conductivity in the head, but variations in the measurements of torso conductivity correlated to breathing and heart activity.

A sensor was developed by Hart et al using a single coil for excitation and detection to measure changes in the brain conductivity for monitoring the progress of brain edema [14]. For edema propagating from a point of trauma to the skull, they proposed placing the coil flat against the head at the trauma site, whereas they proposed surrounding the head with a coil like a headband for brain edema caused by brain stem injury. They derived a theoretical impedance change model and compared this to in vitro impedance measurements made directly using an LCR meter and indirectly with a Colpitts oscillator. In vitro experiments for applications of measuring brain impedance were also conducted by Netz et al using an electromagnetic sensor with a coil configuration akin to that of Tarjan and McFee, but with very small diameter coils [22]. This sensor was mounted to a scanning system with the ultimate goal of being able to scan the whole brain for local changes in conductance indicative of brain edema. It has also been suggested that lung conductivities could be measured with electromagnetic sensors to test for pulmonary edema [12].

Coil sensors have more recently been designed for magnetic induction tomography which could be used for brain imaging and respiratory and cardiac activities. Various coil arrangements have been investigated such as an excitation and one or more receiving coils mounted axially [18][26][33], or an excitation and receiving coils perpendicular to each other [34][16]. Placing the excitation and receiving coils at right angles eliminates any signal from the primary field whereas the other configurations rely on more complex primary field compensation techniques. Multiple sensors are 
arranged in planar arrays or scanning systems to image the distribution of conductivity.

While Hart et al applied their single coil sensor to measuring brain conductivity, other sensors that use only one coil to acts as both the transmitter and receiver have focused on monitoring respiratory and cardiac vital signs. Guardo et al built a sensor to measure cardiac related conductivity changes and tested it in vivo with simultaneous electrocardiogram (ECG) recordings [13]. Their oscillator was fed through an RF voltmeter and they measured the change in amplitude of the signal caused by eddy current losses. They also assessed the impedance range of their sensor in vitro. Their sensor showed good correlation with ECG recordings, but they noted that the sensor was very susceptible to positional changes. They concluded that estimates of blood volume changes in the heart would require estimates of heart location and size.

A related study by Guardo et al assessed the feasibility of monitory heart rate and stroke volume [12]. The sensor was altered with a feedback loop that maintained a constant oscillator amplitude so as to improve the linearity of the sensor. The constant amplitude of the oscillator was controlled by a voltage source which meant that the output of the source was proportional to the conductivity of the body. They related the power dissipated in a conductive sphere to an equivalent resistance in parallel with the coil and derived an equivalent conductance loss. They also proposed a torso model which consisted of nested spheres, all located axially with the coil. They simulated respiratory and cardiac activity using their torso model and analyzed how sensor output would be affected by various parameter changes. They also performed in vivo trials with this sensor and compared and compared ECG signals to their electromagnetic signal. Once again, they concluded that the heart location would need to be known in order to calculate stroke volume, but they suggested that information about heart location, size, and conductivity could possibly be assessed using arrays 
of electromagnetic sensors.

Richer and Adler designed a single coil sensor embedded in a foam mattress [24]. They conducted in vivo trials with the sensor positioned under the lungs to measured respiratory activities. The oscillator was fed through a frequency counter and air flow was measured simultaneously with a pneumotach to follow lung volume changes. The sensor output corresponded well to the pneumotachograph, but frequency changes of the sensor were very small. The sensor also suffered from large motion artifacts.

Most recently, Steffen et al used a single coil sensor to monitor the isolated heart activity during breath holds as well as monitoring the combined lung and heart activity in vivo [29]. They compared using the electromagnetic sensor results they obtained using a capacitive ECG technique. For their electromagnetic analysis they modeled the heart as a disc with a homogeneous induced magnetic field distribution and calculated the induced impedance change in the coil. They used an LC oscillator and the effect of conductivity changes was seen as a frequency shift of their oscillator. They developed an algorithm to cancel the lung activity in order to extract heart signals from their measurements taken during regular breathing. Simultaneous recordings of air flow and pulse plethysmography were compared to the electromagnetic results.

\subsection{Summary}

There are many methods of respiratory monitoring, but the standard techniques that are currently used pose restrictions on the patient. Noncontact sensors are ideal for sleep and apnea monitoring applications in order to observe patients in their natural state. Various electromagnetic sensors have been designed to address this need as well as for other biomedical applications of conductivity measurements.

The electromagnetic sensors for biomedical applications have varied in their coil 
setup and electronic designs. There have been different approaches to theoretically model the behaviour of these sensors. This arises in part because eddy currents are very difficult to calculate directly. Additionally, position and sizes of organs are not constant from one individual to another, and organs have complex shapes and conductivities. There is a lack of validation of the theoretical models developed for these sensors. Furthermore, sensor designs are generally ad-hoc and not based on the electromagnetic models. Eddy current signals are small compared to the driving signals so the changes in frequency measured are relatively small. An approach is therefore needed to optimize the sensitivity of such a sensor. 


\section{Chapter 3}

\section{Theoretical Model}

This chapter presents the theoretical model for the coil sensor. As a person breathes, both the shape and conductivity of the lungs change. We model breathing as a homogeneous change in conductivity over an effected volume. To simplify our analysis, we take the body to be a constant arbitrary shape that contributes a constant background conductivity which we can therefore ignore. We represent the lung as a sphere of conductive material centred above the axis of a single circular coil. Alternate models that could have been used include a conductive cylinder or conductive half space but we chose a sphere as it seemed like the closest anatomical equivalent. The volume of our sphere is set to correspond to the change in volume of the lung.

The electromagnetic analysis follows the same approach as Hart et al [14] but for the geometry of a sphere. The sensitivity of the coil can then be calculated in terms of the change in oscillator frequency caused by the conductive material as seen in Fig. 3.1. We assume that the permeability remains constant at its free space value and the change in permittivity is a secondary effect, so we concentrate on the primary effect of a change in conductivity of our material. This chapter consists of a review of the pertinent electromagnetic theory, a step by step derivation of the theoretical 


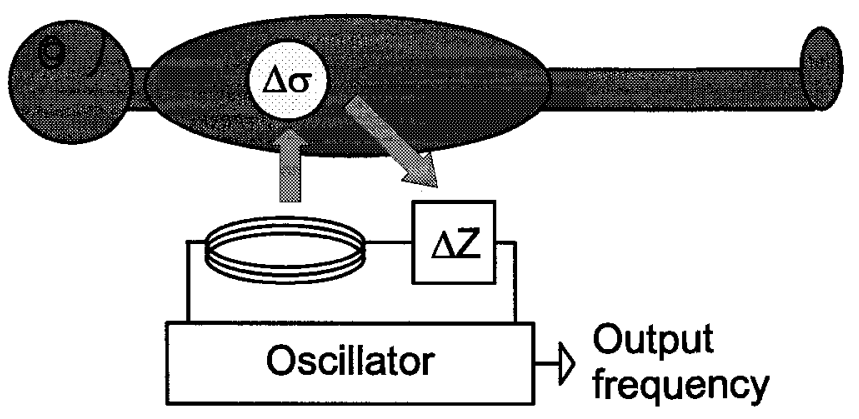

Figure 3.1: We model breathing as a change in conductivity over a spherical volume and ignore the rest of the body. The conductivity change results in a change in impedance that alters the oscillator frequency.

model, a description of how the model is implemented, and the results given by the model.

\subsection{Review of Electrodynamics}

Recall that a current carrying wire produces a magnetic field which circles around the wire according to the right hand rule with a field strength that is inversely proportional to the radius of the circle. Given a current carrying loop of wire, the magnetic field can be calculated by summing the contributions to the field from each segment of the loop using the Biot-Savart law. If the wire is charged with an alternating current, this magnetic field will not be constant. A changing magnetic field will produce an electric field according to Faraday's law. For the geometrical case of a loop of wire carrying an alternating current, the electric field will not be constant. If a conductor is present in this field, eddy currents will be produced within the conductor to compensate for the changes in the electromagnetic field. The current density of the electric field at any given point can be calculated with Ohm's law and the induced eddy current can be calculated by integrating the current density of the field over the area of interest. Each ring of eddy current can be calculated in this manner. This 
brings us back to the original case of a current carrying wire, except that now we have many circular currents that will each provide contributions to a magnetic field. The total magnetic field induced back at the loop of wire can be calculated by summing all the contributions from each of the eddy current loops using the Biot-Savart law once again. Changing the magnetic field inside a loop of wire alters the flux of the loop. This produces an electromagnetic force to counteract the change in flux and this can be seen as either a change in inductance or an effective change in impedance of the coil.

\subsection{Theoretical Model}

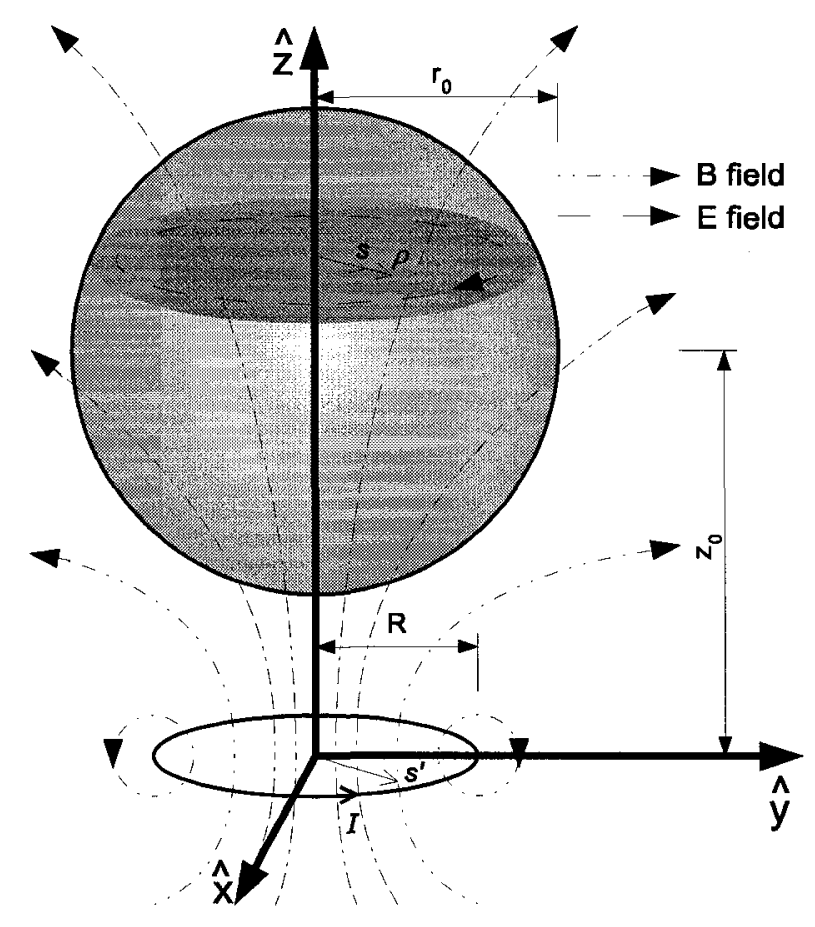

Figure 3.2: Coil carrying current I centred on $\mathrm{x}-\mathrm{y}$ axis with a conductive sphere above it

The theoretical model is derived from quasistatic electromagnetic theory. The 
magnetic field from a coil of $N$ turns, carrying a steady current $I$ centred on the $\mathrm{x}-\mathrm{y}$ axis as seen in Fig. 3.2 can be described by the Biot-Savart law:

$$
\mathbf{B}=\frac{\mu_{0} N I}{4 \pi} \int \frac{d \mathbf{l} \times \hat{\mathbf{r}}}{r^{2}}
$$

where $\mu_{0}=4 \pi \times 10^{-7}\left(N / A^{2}\right)$ is the permeability of free space.

We note that $\hat{\mathbf{r}}=\frac{\mathbf{r}}{|r|}$ and $r^{2}=|r|^{2}$. If the coil has radius $R$, then the magnetic field at a height $z$ above the coil and at a radius $s$ from the $\mathrm{z}$ axis can be solved by making the following substitutions:

$$
\begin{aligned}
d \mathbf{l} & =-R d \phi \sin \phi \hat{\mathbf{x}}+R d \phi \cos \phi \hat{\mathbf{y}} \\
\mathbf{r} & =(s \cos \theta-R \cos \phi) \hat{\mathbf{x}}+(s \sin \theta-R \sin \phi) \hat{\mathbf{y}}+z \hat{\mathbf{z}} \\
|r| & =\left(s^{2}+R^{2}-2 s R \cos (\theta-\phi)+z^{2}\right)^{\frac{1}{2}} .
\end{aligned}
$$

The magnetic field is then given as:

$$
\mathbf{B}(s, z, t)=\frac{\mu_{0} N I}{4 \pi} \int_{0}^{2 \pi} \frac{R z \cos \phi d \phi \hat{\mathbf{x}}+R z \sin \phi d \phi \hat{\mathbf{y}}+\left(R^{2}-R s \cos (\theta-\phi)\right) d \phi \hat{\mathbf{z}}}{\left(s^{2}+R^{2}-2 s R \cos (\theta-\phi)+z^{2}\right)^{\frac{3}{2}}} .
$$

The magnetic field expressed in cylindrical coordinates is then:

$$
\mathbf{B}(s, z, t)=\frac{\mu_{0} N I R}{4 \pi} \int_{0}^{2 \pi} \frac{z d \phi \hat{\mathbf{s}}+(R-s \cos \phi) d \phi \hat{\mathbf{z}}}{\left(s^{2}+R^{2}+z^{2}-2 s R \cos \phi\right)^{\frac{3}{2}}}
$$

where we have noted that $\theta$ is constant and can therefore be dropped. In cylindrical coordinates, $\hat{\mathbf{s}}$ is radial, $\hat{\phi}$ is the angular, and $\hat{\mathbf{z}}$ is equivalent to $\hat{\mathbf{z}}$ in Cartesian coordinates.

Since the radial component of the magnetic field will not contribute to any change in flux in the coil, we are only concerned with the $\hat{\mathbf{z}}$ component which we call $B_{z}(s, z, t)$ 
and we define:

$$
B_{z}(s, z, N, R)=\frac{\mu_{0} N R}{4 \pi} \int_{0}^{2 \pi} \frac{(R-s \cos \phi) d \phi}{\left(s^{2}+R^{2}+z^{2}-2 s R \cos \phi\right)^{\frac{3}{2}}}
$$

where we define $B_{z}(s, z, t)=I B_{z}(s, z, N, R)$. The current and $B_{z}(s, z, t)$ have time dependence, but $B_{z}(s, z, N, R)$ is independent of time. The solution to $B_{z}(s, z, N, R)$ takes the form of elliptic integrals of the first and second kind.

The electric field at the loop of radius $s$ and height $z$ can then be calculated from the flux $\Phi$ through the surface of the loop using the integral form of Faraday's Law:

$$
\begin{aligned}
\oint \mathbf{E} \cdot d \mathbf{l} & =-\frac{d \Phi}{d t} \\
& =-\int \frac{d \mathbf{B}}{d t} \cdot d \mathbf{a}
\end{aligned}
$$

and the electric field at a point with radius $s$ and height $z$ is then:

$$
\begin{aligned}
\mathbf{E}(\rho, z, t) & =-\frac{1}{2 \pi \rho} \frac{d I}{d t} \int_{0}^{\rho} \int_{0}^{2 \pi} s B_{z}(s, z, N, R) d \phi d s \hat{\phi} \\
& =-\frac{1}{\rho} \frac{d I}{d t} \int_{0}^{\rho} s B_{z}(s, z, N, R) d s \hat{\phi} .
\end{aligned}
$$

We apply Ohm's Law to find the current density at this point:

$$
\begin{aligned}
\mathbf{J}(\rho, z, t) & =(\sigma+j \omega \epsilon) \mathbf{E}(\rho, z, t) \\
& =-\frac{(\sigma+j \omega \epsilon)}{\rho} \frac{d I}{d t} \int_{0}^{\rho} s B_{z}(s, z, N, R) d s \hat{\phi}
\end{aligned}
$$

where $\sigma$ is the conductivity and $\epsilon$ is the permittivity. The permittivity of free space is $\epsilon_{0}=8.85 \times 10^{-12} \frac{C^{2}}{N m^{2}}$. This calculation uses the assumption that the material has isotropic and homogeneous conductivity and permittivity so that $\mathbf{E} \| \mathbf{J}$. 


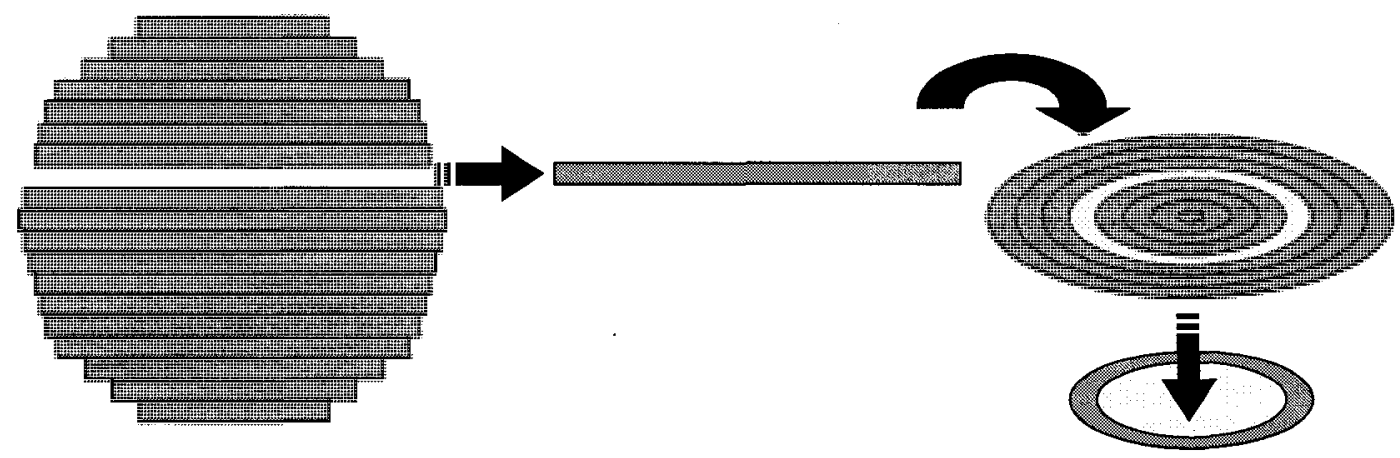

Figure 3.3: The sphere is divided into many current carrying loops

The induced eddy current through a surface is then found by integrating the current density over the area of interest $\mathbf{I}=\int \mathbf{J} \cdot d \mathbf{a}$. We want to model our conductive material as a sphere and do this by breaking the sphere into very thin disks, which are parallel to the coil, and summing the contributions from each of the disks. Each of those conductive disks, in turn, can be modeled as concentric eddy current carrying loops which we sum over the radius of the disk as seen in Fig. 3.3. We describe an element of eddy current within the sphere in differential form:

$$
d \mathbf{I}(\rho, z, t)=-(\sigma+j \omega \epsilon) \frac{d I}{d t} d z \frac{d \rho}{\rho} \int_{0}^{\rho} s B_{z}(s, z, N, R) d s \hat{\phi} .
$$

We now project backwards and calculate the magnetic field induced at the plane of the coil by each element of eddy current. Once again, we only care about the component in the axial direction. The $\hat{\mathbf{z}}$ component of the induced magnetic field in the $\mathrm{x}$-y plane at a radius $s^{\prime}$ is then:

$$
\begin{aligned}
B_{z}\left(s^{\prime}, z, t\right) & =\int d \mathbf{I}(\rho, z, t) B_{z}\left(s^{\prime}, z, 1, \rho\right) \\
& =-(\sigma+j \omega \epsilon) \frac{d I}{d t} \int_{z_{1}}^{z_{2}} \int_{0}^{r_{z}} \int_{0}^{\rho} \frac{s}{\rho} B_{z}(s, z, N, R) B_{z}\left(s^{\prime}, z, 1, \rho\right) d s d \rho d z
\end{aligned}
$$


where $B_{z}\left(s^{\prime}, z, 1, \rho\right)$ is our original $B_{z}(s, z, N, R)$ expression from Eqn. 3.7 is used with the following substitutions: $R=\rho, s=s^{\prime}$ and $N=1$.

We define the radial limit of each disk as:

$$
r_{z}=\sqrt{r_{0}^{2}-\left(z_{0}-z\right)^{2}}
$$

where $z_{0}$ is the height of the centre of the sphere above the coil and $r_{0}$ is the radius of the sphere, and we sum the contributions from each disk by integrating over the bounds:

$$
\begin{aligned}
& z_{1}=z_{0}-r_{0} \\
& z_{2}=z_{0}+r_{0} .
\end{aligned}
$$

If we were to look at the less geometrically complex case of a cylinder, the radial limit and the bounds would be constants; the radial limit would simply be the radius of the cylinder, the lower bound would be the height of the base of the cylinder, and the upper bound would be the height of the top of the cylinder.

Returning to the case of a conductive sphere broken into disks, the magnetic field induced at the coil causes a change in the magnetic flux inside a loop of the coil given by:

$$
\begin{aligned}
\Phi & =\int B_{z}\left(s^{\prime}, z, t\right) \cdot d \mathbf{a} \\
& =\int_{0}^{R} \int_{0}^{2 \pi} s^{\prime} B_{z}\left(s^{\prime}, z, t\right) d \phi d s^{\prime} \\
& =-2 \pi(\sigma+j \omega \epsilon) \frac{d I}{d t} \int_{0}^{R} \int_{z_{1}}^{z_{2}} \int_{0}^{r_{z}} \int_{0}^{\rho} \frac{s s^{\prime}}{\rho} B_{z}(s, z, N, R) B_{z}\left(s^{\prime}, z, 1, \rho\right) d s d \rho d z d s^{\prime} \hat{\mathbf{z}}
\end{aligned}
$$


where we recall $R$ is the radius of the coil. The change in flux manifests as an electromotive force (EMF) proportional to the number of loops in the current carrying coil:

$$
\begin{aligned}
\varepsilon & =-N \frac{d \Phi}{d t} \\
& =2 \pi N(\sigma+j \omega \epsilon) \frac{d^{2} I}{d t^{2}} \int_{0}^{R} \int_{z_{1}}^{z_{2}} \int_{0}^{r_{z}} \int_{0}^{\rho} \frac{s s^{\prime}}{\rho} B_{z}(s, z, N, R) B_{z}\left(s^{\prime}, z, 1, \rho\right) d s d \rho d z d s^{\prime} \hat{\phi}
\end{aligned}
$$

We assume that the current is sinusoidal and given by $I=A e^{j \omega t}$. As such, the second derivative of current is $-\omega^{2} I$ so our EMF expression simplifies to:

$$
\varepsilon=-2 \pi \omega^{2} N I(\sigma+j \omega \epsilon) \int_{0}^{R} \int_{z_{1}}^{z_{2}} \int_{0}^{r_{z}} \int_{0}^{\rho} \frac{s s^{\prime}}{\rho} B_{z}(s, z, N, R) B_{z}\left(s^{\prime}, z, 1, \rho\right) d s d \rho d z d s^{\prime} \hat{\phi}
$$

The change in the inductance of the coil is:

$$
\begin{aligned}
L & =\frac{N \Phi}{I} \\
& =-\frac{\varepsilon}{j \omega I} .
\end{aligned}
$$

Alternatively, the EMF can be seen by the coil as an effective series impedance:

$$
\begin{aligned}
Z & =-\frac{\varepsilon}{I} \\
& =2 \pi \omega^{2} N(\sigma+j \omega \epsilon) \int_{0}^{R} \int_{z_{1}}^{z_{2}} \int_{0}^{r_{z}} \int_{0}^{\rho} \frac{s s^{\prime}}{\rho} B_{z}(s, z, N, R) B_{z}\left(s^{\prime}, z, 1, \rho\right) d s d \rho d z d s^{\prime} \hat{\phi}
\end{aligned}
$$

Eqn. 3.29 gives the effective series impedance that will result from sphere of a certain conductivity centred above the axis of the coil. We are interested in the change in 
impedance caused by a change in conductivity within that sphere. We calculate that the change in conductivity we expect from an inflated versus deflated lung is more than ten times the change caused by the change in permittivity. This calculation was based on numbers obtained from an on-line resource [2] for dielectric properties of tissues that calculates values using the parametric model and results of [8][9][10][11]. Since there is a relatively small change due to permittivity differences, we concentrate on the primary effect caused by the change in conductivity and conclude that the effective series impedance has the following relationship:

$$
\Delta Z \propto \omega^{2} N^{2} \Delta \sigma F_{c o i l}(R)
$$

where $\omega$ is the angular frequency of the oscillator, $N$ is the number of loops in the coil, $\Delta \sigma$ is the change in the conductivity, and $F_{\text {coil }}(R)$ is some non-linear function of the coil radius $R$. The impedance is proportional to the square of the number of loops $N$ because there is an additional factor of $\mathrm{N}$ in the formula for $B_{z}(s, z, N, R)$ from Eqn. 3.7. The number of loops influences the inductance of the coil which affects the frequency of the oscillator.

We calculate the theoretically induced impedance for different coil radii and scale the results to be independent of the frequency, number of turns, and conductivity. Fig. 3.4 shows the output where we have taken the conductive sphere to be $2.5 \mathrm{~L}$ and the distance from the coil to the centre of the sphere to be $10 \mathrm{~cm}$.

\subsection{Implementation of Theoretical Model}

In order to solve Eqn. 3.29 it is necessary to integrate numerically. This was solved using Matlab by first integrating Eqn. 3.7 for $B_{z}(s, z, N, R)$ symbolically and then 


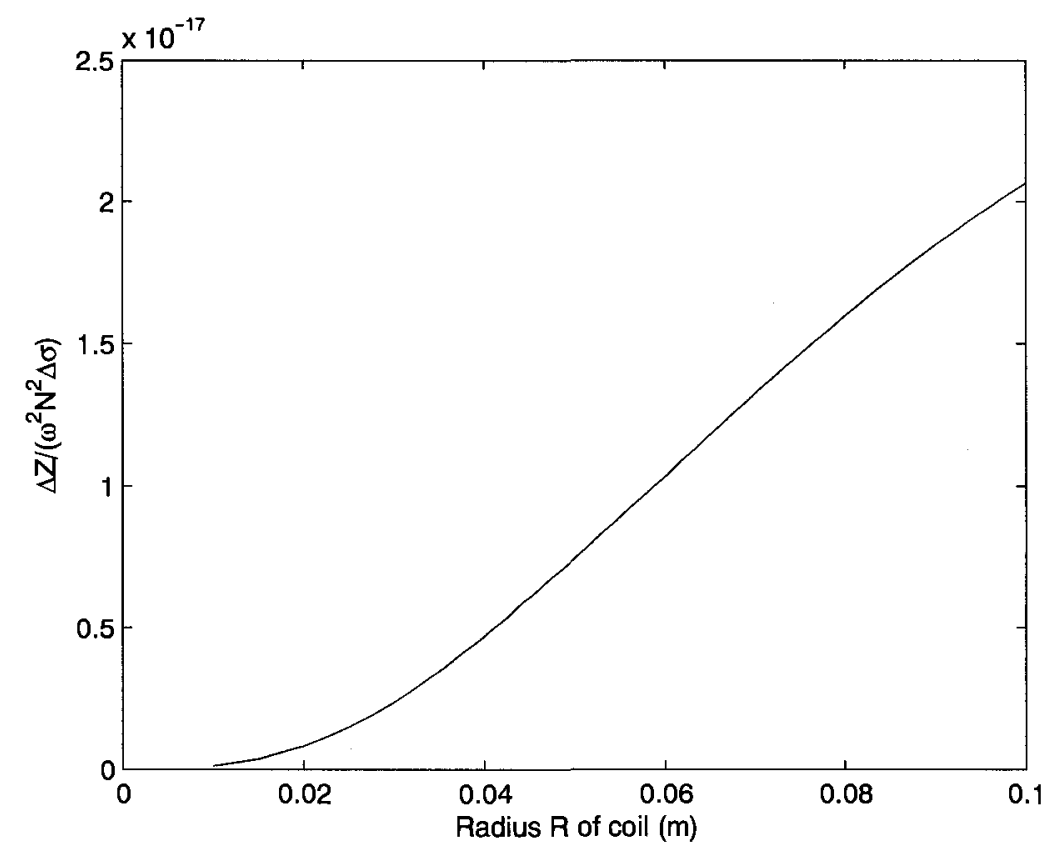

Figure 3.4: The relationship between the scaled theoretical impedance factor and the coil radius $\mathrm{R}$

performing the rest of the integration numerically using adaptive Simpson quadrature by nesting functions as follows:

$$
\begin{aligned}
\text { Impedance } & =-\omega^{2} N \cdot \text { Flux } \\
\text { Flux } & =\int_{0}^{R} \int_{0}^{2 \pi} s^{\prime} \cdot B_{\text {Induced }}\left(s^{\prime}\right) d \phi d s^{\prime} \\
B_{\text {Induced }}\left(s^{\prime}\right) & =\int_{z_{1}}^{z_{2}} \int_{0}^{r_{z}} B\left(s^{\prime}, z, \rho, 1\right) \cdot J(\rho, z) d \rho d z \\
J(\rho, z) & =(\sigma+j \omega \epsilon) \cdot E_{\text {Induced }}(\rho, z) \\
E_{\text {Induced }}(\rho, z) & =-\frac{1}{2 \pi \rho} \int_{0}^{\rho} \int_{0}^{2 \pi} s \cdot B(s, z, R, N) d \phi d s \\
B(s, z, R, N) & =B_{z}(s, z, N, R) \text { in elliptic integral form }
\end{aligned}
$$

In the implementation of this algorithm, we divide the double integral of the flux into a nested function as well. The Matlab code for the model is included in Appendix A. 
To simplify the phantom trials, we used a cylindrical phantom, so a cylindrical conductor model was used to compare the theoretical predictions to the measured results. The cylindrical conductor model had different radial limits and bounds as discussed in the derivation, but otherwise it followed the same approach as the spherical model. The spherical conductor model was used for the in vivo trials. For the in vivo experiments, we can model breathing by modeling the lung as a sphere, which expands on inspiration. We say that the difference in conductivity of a deflated versus inflated lung is $\Delta \sigma=\sigma_{d}-\sigma_{i}=0.21 \mathrm{~S} / \mathrm{m}$ at the average operating frequencies of our in vivo trials whereas $\omega \Delta \epsilon=0.0176$ and 0.0171 . These calculations were made based on numbers obtained from an on-line resource [2] for dielectric properties of tissues that calculates values using the parametric model and results of [8][9][10][11].

The radius of the sphere is given by,

$$
r_{0}=\sqrt[3]{\frac{3 V}{4000 \pi}}
$$

where $V$ is the volume of inspired air of one lung in litres. We estimate a total lung capacity breathing manoeuver to be 2.5 litres for one lung, which corresponds to a radius $r_{0}=0.0842 \mathrm{~m}$. The second breathing manoeuver in our in vivo trials consists of breathing in one litre of air (500 mL per lung), which corresponds to a spherical conductor radius of $r_{0}=0.0492 \mathrm{~m}$ for one lung. The final breathing manoeuver involves breathing $400 \mathrm{~mL}$ of air (200 mL per lung), which corresponds to $r_{0}=0.0363 \mathrm{~m}$ per lung. We estimate that the thorax is 20 to $24 \mathrm{~cm}$ thick and estimate from this that the sphere is centred about $10 \mathrm{~cm}$ above the coil.

Table 3.1 gives the theoretical change in impedance we predict for the in vivo trials as a function of oscillator frequency $f$ and the number of turns in the coil $N$. We use the sphere radii for breathing volumes and the distance between coil and centre 


\begin{tabular}{|l|c|c|c|}
\hline & $\mathbf{R}=\mathbf{3 . 7} \mathbf{~ c m}$ & $\mathbf{R = 4} \mathbf{~ c m}$ & $\mathbf{R = 4 . 6} \mathbf{c m}$ \\
\hline TLC & $3.26 \cdot 10^{-17} f^{2} N^{2}$ & $3.89 \cdot 10^{-17} f^{2} N^{2}$ & $5.24 \cdot 10^{-17} f^{2} N^{2}$ \\
\hline 1 Litre & $1.95 \cdot 10^{-18} f^{2} N^{2}$ & $2.42 \cdot 10^{-18} f^{2} N^{2}$ & $3.53 \cdot 10^{-18} f^{2} N^{2}$ \\
\hline 400 $\mathbf{~ m L}$ & $5.09 \cdot 10^{-19} f^{2} N^{2}$ & $6.41 \cdot 10^{-19} f^{2} N^{2}$ & $9.59 \cdot 10^{-19} f^{2} N^{2}$ \\
\hline
\end{tabular}

Table 3.1: The theoretical change in impedance $\Delta Z$ that our model predicts for three breathing volumes using three different coil radii R. Results are given in terms of oscillator frequency $f$ and the number of turns $N$ of the coil

of lung as listed above. We use the change in conductivity between a deflated and inflated lung $\Delta \sigma=0.21 \mathrm{~S} / \mathrm{m}$ for all three volume cases. We make the calculations for three coil radii which are chosen based on the cylinder molds that we have for constructing coils.

\subsection{Summary}

This chapter explored the theoretical effect of a conductive object placed near a current carrying coil and we established a model based on this effect. A model of the change in impedance was derived as a function of coil and object geometries. Based on this effect, we derived a proportionality formula that can be used to optimize sensor components. Finally, we outlined the approach for implementing our model and provided theoretical predictions of sensor behaviour. 


\section{Chapter 4}

\section{Electronic Design}

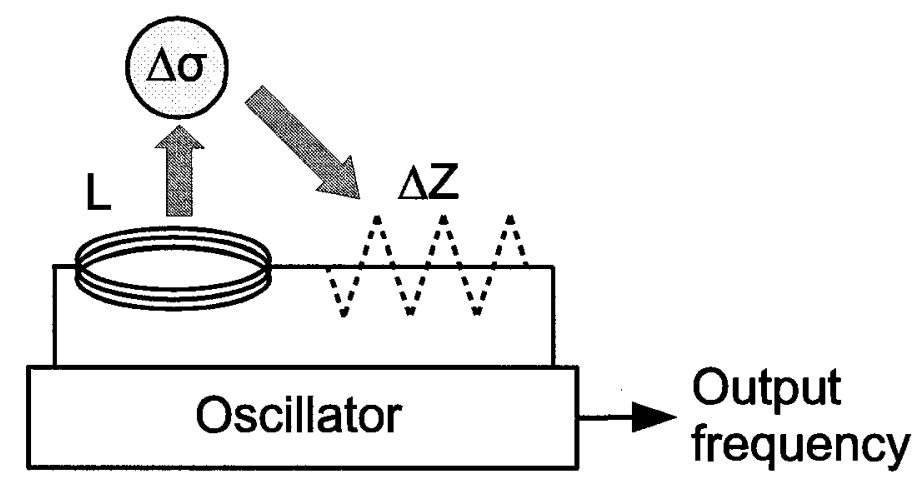

Figure 4.1: Block diagram of the basic functionality of the oscillator

The electromagnetic coil sensor requires circuitry whereby the output is changed by changing an inductive component. When the coil is exposed to a change in conductivity in its surroundings, an effective series impedance $\Delta Z$ is added and this changes the oscillating frequency as seen in Fig. 4.1. Several fields in engineering require accurate measurements of frequency. For example, very small changes in the resonance frequency of the system can be used for contactless mapping of a surface in the atomic force microscopy field [1]. We examined LC Colpitts oscillators and a CMOS oscillator as potential circuits. After testing several circuit designs, one of 
each type of oscillator was chosen for the experiments. The circuit components were then selected to maximize the output of the sensor. This chapter presents the circuits that were investigated and the component selection process.

\subsection{Review of Oscillators}

Oscillators are an essential tool in electronics as they are needed for signal generation and timing mechanisms. The basic requirements for an oscillator are a power source, frequency determining components, amplification, and positive feedback [19]. For our electromagnetic sensor application, an inductor must be part of the frequency determining components.
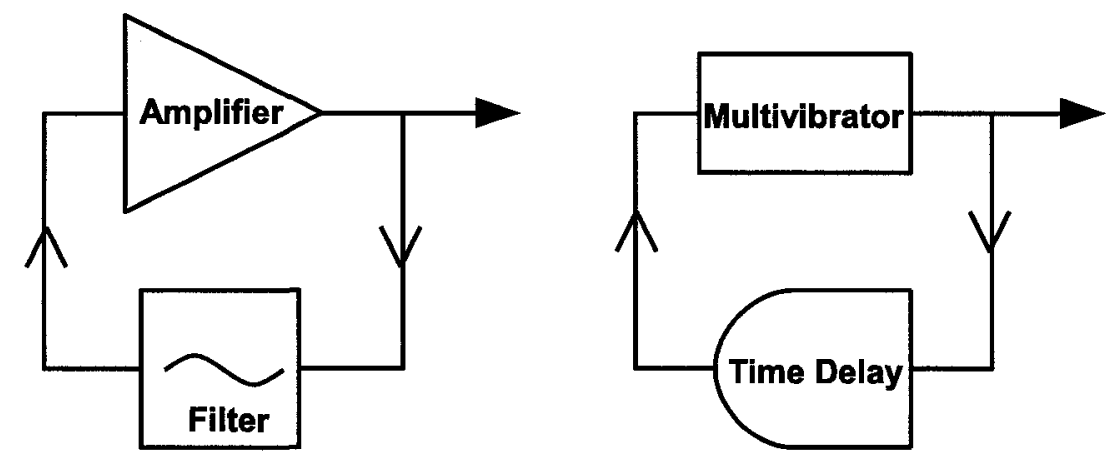

Figure 4.2: Block diagrams for a harmonic oscillator and a relaxation oscillator

The first type of oscillator circuit investigated for the electromagnetic coil sensor was a Colpitts oscillator. A Colpitts oscillator is an LC harmonic oscillator and it produces sine wave signals. Harmonic oscillators amplify a signal, feed it through a filter, and feed it back through the amplifier. The second type of oscillator examined was a relaxation oscillator which produced a square wave signal. A relaxation oscillator relies on a threshold device such as a multivibrator or astable latch to switch rapidly between two states and a time delay element to control the frequency. Fig. 4.2 shows the basic components of a harmonic oscillator and a relaxation oscillator. The 
relaxation oscillator circuit that was used was inspired by a design that employed a series of CMOS inverters connected via an RC network [5]. In this case, the inverters were the threshold devices and the RC network provided the time delay. This circuit was altered so that an inductor was used rather than a capacitor for the delay.

\subsection{Colpitts Oscillator}

Three different Colpitts designs were built and tested, each using a different component as the active element.

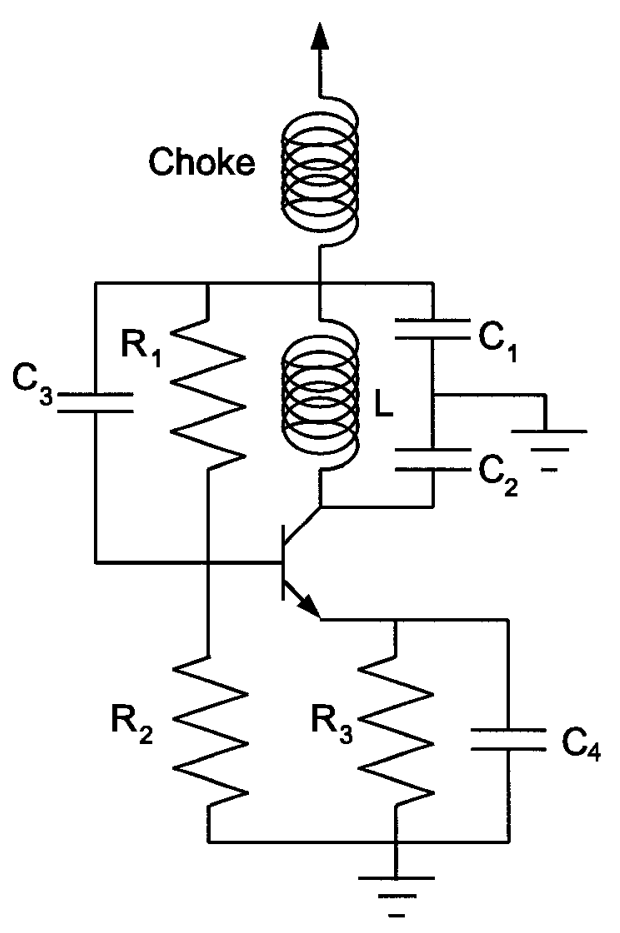

Figure 4.3: A Colpitts oscillator with a bipolar junction transistor (using $L=65 \mu \mathrm{H}$, $C_{1}=C_{2}=100 \mathrm{pF}, C_{3}=C_{4}=10 \mathrm{nF}, R_{1}=10 \mathrm{k} \Omega, R_{2}=5 \mathrm{k} \Omega, R_{3}=150 \Omega$, and Choke $=33 \mu \mathrm{H}$, f was $2.8 \mathrm{MHz}$ )

Fig. 4.3 shows the first design from [27], which was implemented with a 2N3904 bipolar junction transistor. The second design, shown in Fig. 4.4, used an LM 6181 current feedback operational amplifier (op-amp). This design was obtained from an 


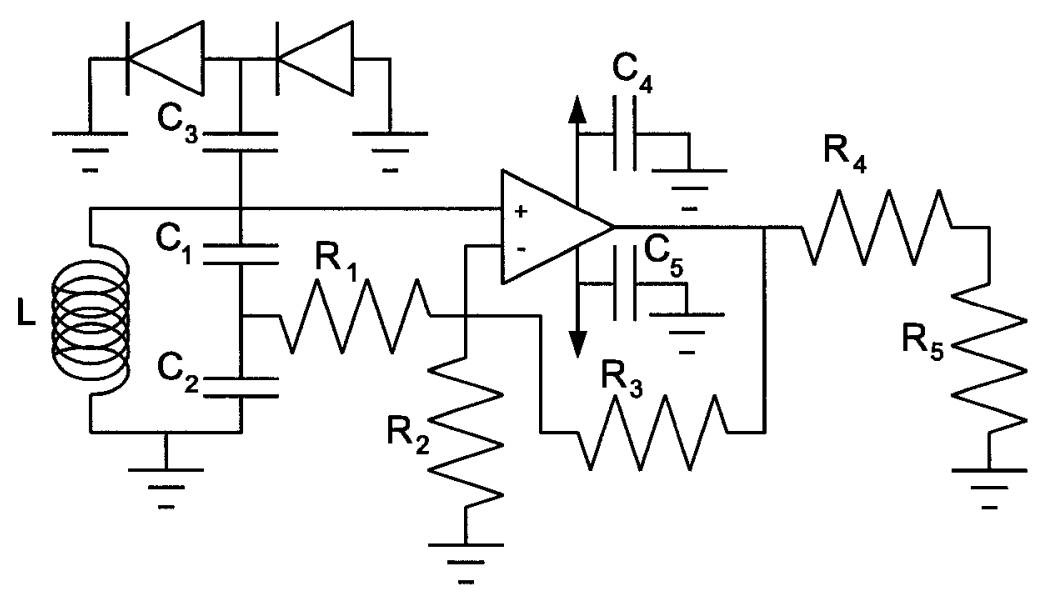

Figure 4.4: A Colpitts oscillator with a current feedback operational amplifier (using 1 N4148 diodes, $L=108 \mu H, C_{1}=C_{2}=10 p F, C_{3}=C_{4}=C_{5}=100 n F, R_{1}=330 \Omega$, $R_{2}=100 \Omega, R_{3}=1 k \Omega$, and $R_{4}=R_{5}=50 \Omega$, f was $4.1 \mathrm{MHz}$ )

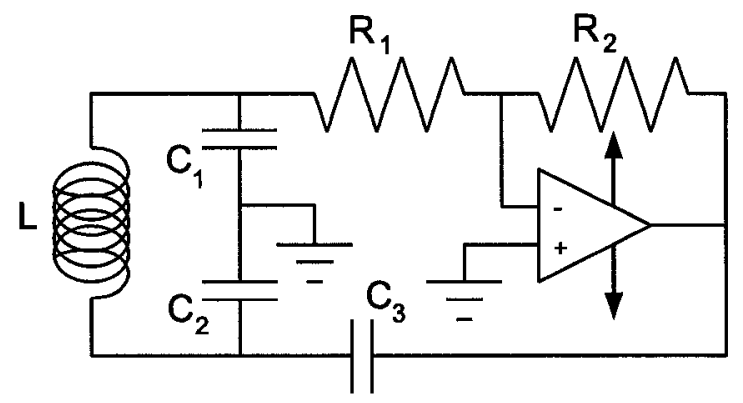

Figure 4.5: A Colpitts oscillator with a general purpose operational amplifier 
EDN publication [17]. Finally, a basic design that utilized a general purpose op-amp was tried [19]. This circuit, shown in Fig. 4.5, was realized with an LM318 op-amp.

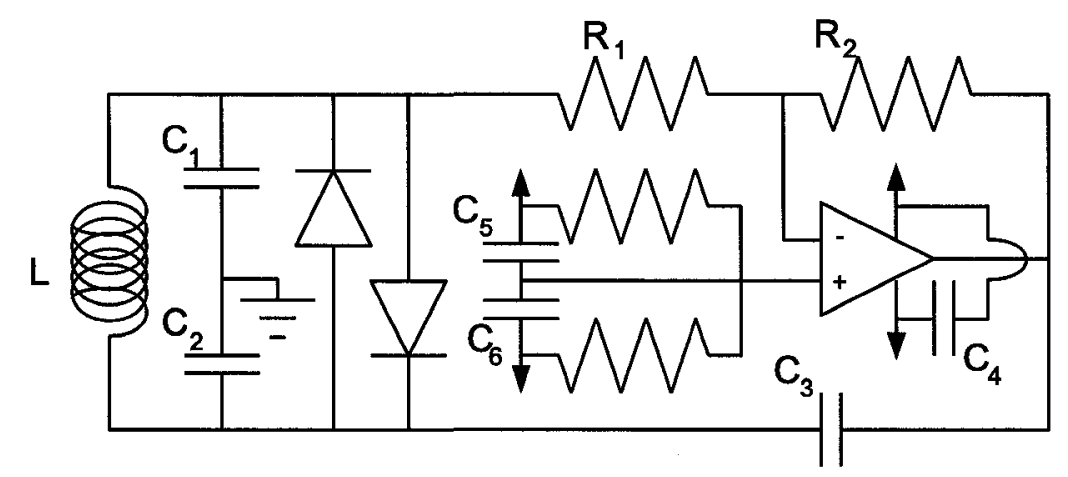

Figure 4.6: The Colpitts oscillator circuit design that was chosen

The general purpose op-amp design was chosen over the first two because it demonstrated the best consistency: it output the cleanest wave shape, and its simplicity made it easy to implement. The original circuit was changed slightly to the final version, shown in Fig. 4.6. The non-inverting input of the op-amp is fed into a resistor and capacitor network so that a single power supply could be used. The 1N4148 diodes were added across the inductor as limiters in order to provide amplitude stabilization of the signal.

\subsubsection{Frequency}

The Colpitts oscillator is characterized by its tank circuit, which contains an inductor in parallel with two capacitors. Refer to Fig. 4.5 for the basic Colpitts circuit with an operational amplifier as the active element. The resonance frequency can be derived by replacing the op-amp with a controlled source model as seen in Fig. 4.7. Assuming the op-amp is ideal, the input current is zero, so

$$
\frac{v_{1}-v_{-}}{R_{1}}=\frac{v_{-}-v_{0}}{R_{2}}
$$




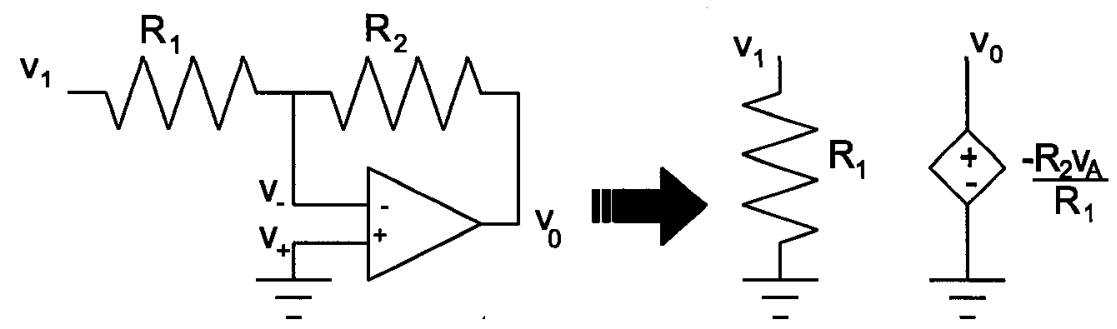

Figure 4.7: The controlled source model for an ideal op-amp

There is a virtual ground at $v_{-}$since $v_{+}$is grounded and the output voltage is given by

$$
v_{0}=-\frac{R_{2}}{R_{1}} v_{1} .
$$

The op-amp is thus replaced with a voltage controlled voltage source and the circuit

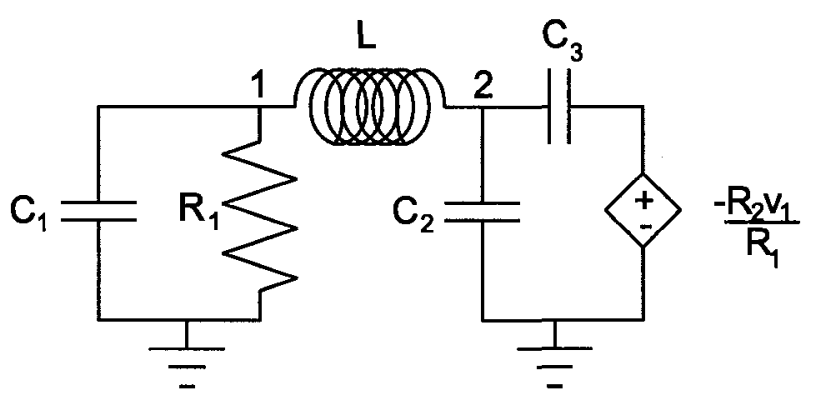

Figure 4.8: The Colpitts op-amp circuit with the controlled source model

is rearranged to look like Fig. 4.8. Performing nodal analysis at 1 yields

$$
\begin{aligned}
0 & =j \omega C_{1} v_{1}+\frac{v_{1}}{R_{1}}+\frac{v_{1}-v_{2}}{j \omega L} \\
\frac{v_{2}-v_{1}}{j \omega L} & =j \omega C_{1} v_{1}+\frac{v_{1}}{R_{1}} \\
v_{2} & =v_{1}\left(1-\omega^{2} C_{1} L+\frac{j \omega L}{R_{1}}\right),
\end{aligned}
$$


Where $v_{1}$ and $v_{2}$ are the voltages at nodes 1 and 2 respectively. Using nodal analysis at 2 and substituting Eqn. 4.4 and Eqn. 4.5 gives

$$
\begin{aligned}
0 & =j \omega C_{2} v_{2}+j \omega C_{3}\left(v_{2}+\frac{R_{2}}{R_{1}} v_{1}\right)+\frac{v_{2}-v_{1}}{j \omega L} \\
0 & =\frac{1}{R_{1}}-\omega^{2} \frac{L}{R_{1}}\left(C_{2}+C_{3}\right)+j \omega\left(C_{1}+C_{2}+C_{3}\right)-j \omega^{3} C_{1} L\left(C_{2}+C_{3}\right)+j \omega C_{3} \frac{R_{2}}{\left.R_{1} .7\right)}
\end{aligned}
$$

For this to be true, both the real and imaginary parts must be zero. We look first at the imaginary part and find that

$$
\omega=\sqrt{\frac{C_{1}+C_{2}+C_{3} \frac{R_{1}+R_{2}}{R_{1}}}{C_{1} L\left(C_{2}+C_{3}\right)}},
$$

but $C_{3}$ is very small, so we say that the resonant frequency is given by the tank circuit:

$$
\omega_{0} \simeq \frac{1}{\sqrt{L \frac{C_{1} C_{2}}{C_{1}+C_{2}}}} \simeq \frac{1}{\sqrt{L C_{T}}}
$$

where $C_{T}$ is the total capacitance from the series combination of $C_{1}$ and $C_{2}$. Looking now at the real part of Eqn. 4.7 and substituting Eqn. 4.8, we find the condition for oscillations to be sustained,

$$
\frac{C_{2}}{C_{3}}=-\frac{R_{1}+R_{2}}{R_{1}}
$$

Clearly Eqn. 4.10 is impossible for real resistance and capacitance values. Instead, the gain required for oscillation is determined by the phase delay in the amplifier which contributes a signal out of phase.

When the coil is exposed to a change in the conductivity of its surroundings, we model the change in the impedance of the coil, $\Delta Z$, as a series resistance, as shown in Fig. 4.9. The added impedance changes our nodal analysis at 1 and we get the 


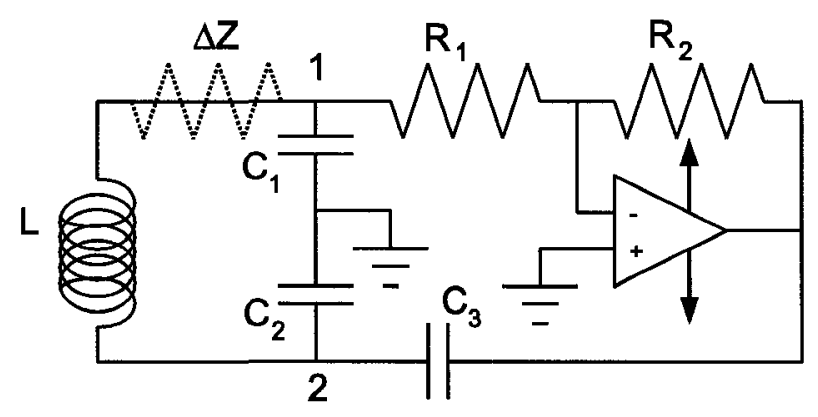

Figure 4.9: The Colpitts op-amp circuit with the impedance effect from a change in conductivity modeled as a dashed resistor

following:

$$
\begin{aligned}
0 & =j \omega C_{1} v_{1}+\frac{v_{1}}{R_{1}}+\frac{v_{1}-v_{2}}{j \omega L+\Delta Z} \\
\frac{v_{2}-v_{1}}{j \omega L+\Delta Z} & =j \omega C_{1} v_{1}+\frac{v_{1}}{R_{1}} \\
v_{2} & =v_{1}\left(1+\frac{\Delta Z}{R_{1}}-\omega^{2} C_{1} L+\frac{j \omega L}{R_{1}}+j \omega \Delta Z C_{1}\right),
\end{aligned}
$$

and at 2 ,

$$
\begin{aligned}
0= & j \omega C_{2} v_{2}+j \omega C_{3}\left(v_{2}+\frac{R_{2}}{R_{1}} v_{1}\right)+\frac{v_{2}-v_{1}}{j \omega L+\Delta Z} \\
0= & \frac{1}{R_{1}}-\omega^{2}\left(C_{1}+C_{3}\right)\left(\frac{L}{R_{1}}+\Delta Z C_{1}\right)+j \omega\left(C_{1}+C_{2}+C_{3}\right) \\
& -j \omega^{3} C_{1} L\left(C_{2}+C_{3}\right)+\frac{j \omega}{R_{1}}\left(\Delta Z C_{2}+\Delta Z C_{3}+R_{2} C_{3}\right) .
\end{aligned}
$$

The resonance frequency is then

$$
\begin{aligned}
& \omega_{\Delta Z}=\sqrt{\frac{C_{1}+C_{2} \frac{R_{1}+\Delta Z}{R_{1}}+C_{3} \frac{R_{1}+R_{2}+\Delta Z}{R_{1}}}{C_{1} L\left(C_{2}+C_{3}\right)}} \\
& \omega_{\Delta Z} \simeq \frac{1}{\sqrt{L \frac{C_{1} C_{2}}{C_{1}+C_{2} \frac{R_{1}+\Delta Z}{R_{1}}}}} .
\end{aligned}
$$


We will choose $C_{1}=C_{2}=C$ and we will calculate our relative change in frequency parameter, which is what we want to optimize for our circuit design.

$$
\begin{aligned}
\frac{\Delta \omega}{\omega} & =\frac{\omega_{\Delta Z}-\omega_{0}}{\omega_{0}} \\
& \simeq \frac{\sqrt{\frac{2+\frac{\Delta Z}{R_{1}}}{L C}}-\sqrt{\frac{2}{L C}}}{\sqrt{\frac{2}{L C}}} \\
& \simeq \sqrt{1+\frac{\Delta Z}{2 R_{1}}}-1 \\
& \simeq \frac{\Delta Z}{4 R_{1}},
\end{aligned}
$$

where we have used the Taylor Series approximation for the square root.

\subsubsection{Circuit Optimization}

From Eqn. 4.21 we see that we should maximize $\Delta Z$ and minimize $R_{1}$. We derived

a proportionality formula for $\Delta Z$ in the last chapter at Eqn. 3.30, and we know the resonance frequency $\omega_{0}$ from Eqn. 4.9. We will use the theoretical inductance of a multilayer air core coil,

$$
L=\frac{31.5 \cdot 10^{-6} R^{2} N^{2}}{6 R+9 l+10 d},
$$

where $R$ is the average radius, $N$ is the number of turns, $l$ is the length, and $d$ is the thickness (the difference between the outer and inner radius) [35]. We reevaluate our relative change in frequency parameter by substituting these equations back into 
Eqn. 4.21,

$$
\begin{aligned}
\frac{\Delta \omega}{\omega} & \propto \frac{\omega^{2} N^{2} \Delta \sigma F_{\text {coil }}(R)}{R_{1}} \\
\propto & \frac{N^{2} \Delta \sigma F_{\text {coil }}(R)}{L C_{T} R_{1}} \\
\propto & \frac{(6 R+9 l+10 d) \Delta \sigma F_{\text {coil }}(R)}{R^{2} C_{T} R_{1}},
\end{aligned}
$$

where we recall that $F_{\text {coil }}(R)$ is a nonlinear function of the coil radius. Consequently, to optimize the sensitivity of this circuit, we want to maximize the coil length and thickness, and minimize the resistor $R_{1}$ and tank capacitors. Since the coil length and diameter are functions of the number of turns, this means that we want to maximize $N$. Simulations of the coil radius function in the range $R=0.01$ to $R=0.10 \mathrm{~m}$ show that $\frac{F_{\text {coil }}(R)}{R}$ increases, but starts to plateau at $R \simeq 0.1 \mathrm{~m}$ and $\frac{F_{\text {coil }}(R)}{R^{2}}$ peaks at $R \simeq 0.045 \mathrm{~m}$.

\subsection{Relaxation Oscillator}

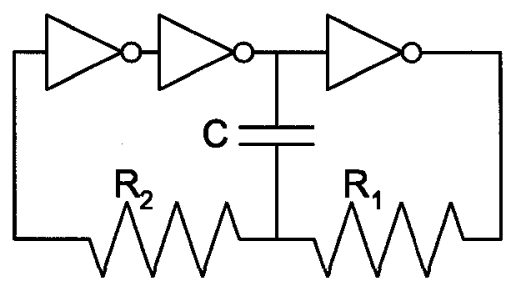

Figure 4.10: The original relaxation oscillator design with a capacitor for the time delay [5]

As stated earlier, the relaxation circuit was adapted from a design that used CMOS inverters as the threshold devices. Any odd number of inverters connected in series with feedback will oscillate and produce a square wave as the output switches between high and low. The original circuit is shown in Fig. 4.10 [5]. Rather than using 


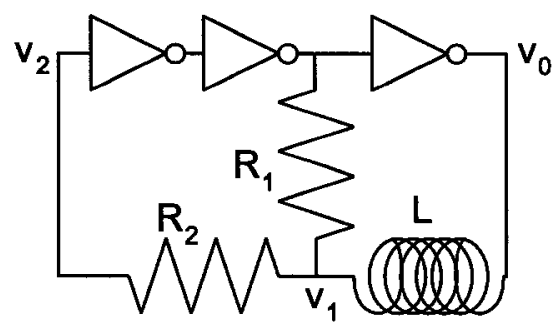

Figure 4.11: Our modified relaxation oscillator design with an inductor for the time delay

a capacitor to connect the three inverters, our circuit substituted an inductor into the feedback network as shown in Fig. 4.11. We used three inverters from a 74HC04 Hex Inverter chip to build this circuit and the inductor and resistors provides a time delay that determines the frequency of the relaxation circuit.

\subsubsection{Frequency}
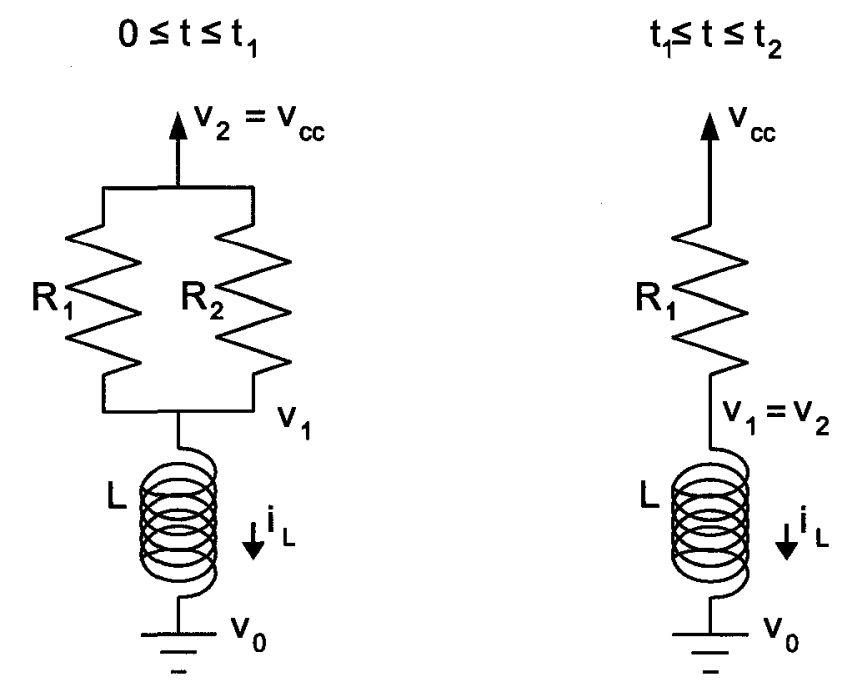

Figure 4.12: The relaxation circuit simplifies for two time interval cases

If we assume that at time $t=0$ the output voltage switches from high to low, we can simplify the circuit from Fig. 4.11 to the two cases shown in Fig. 4.12 for different 
time intervals. We can therefor describe the voltage at $v_{1}$ as follows:

$$
\begin{aligned}
v_{1} & =v_{c c}\left(\frac{2 R_{1}+3 R_{2}}{2 R_{1}+2 R_{2}}\right) e^{\left(\frac{-R_{1} \| R_{2}}{L}\right) t} \text { for } 0 \leq t \leq t_{1} \\
& =v_{c c} e^{\frac{-R_{1}}{L}\left(t-t_{1}\right)} \text { for } t_{1} \leq t \leq t_{2} \\
& =\frac{v_{c c}}{2} \text { for } t=t_{2},
\end{aligned}
$$

where $v_{c c}$ is the power supply voltage.

When the voltage $v_{1}$ reaches the threshold voltage at $t_{2}$, the inverters switch. This marks half of the period at

$$
\begin{aligned}
t_{2} & =\frac{L \ln 2}{R_{1}}+t_{1} \\
t_{1} & =\frac{L \ln \left(\frac{2 R_{1}+3 R_{2}}{2 R_{1}+2 R_{2}}\right)}{R_{1} \| R_{2}}
\end{aligned}
$$

and the frequency of the oscillator is then,

$$
\begin{aligned}
f & =\frac{1}{2 t_{2}} \\
& =\frac{R_{1}}{2 L\left[\ln 2+\left(\frac{R_{1}+R_{2}}{R_{2}}\right) \ln \left(\frac{2 R_{1}+3 R_{2}}{2 R_{1}+2 R_{2}}\right)\right]}
\end{aligned}
$$

We can simplify this for three special cases of resistor choice:

$$
\begin{aligned}
& \text { if } R_{1} \gg R_{2}, f \simeq \frac{R_{1}}{2 L \ln 3.3}, \\
& \text { if } R_{1}=R_{2}, f=\frac{R_{1}}{2 L \ln \frac{25}{8}}, \\
& \text { if } R_{1} \ll R_{2}, f \simeq \frac{R_{1}}{2 L \ln 3} .
\end{aligned}
$$

The timing diagram of the voltage and current in the relaxation circuit is illustrated in Fig. 4.13. The current $i_{L}$ through the coil ranges from $\sim \frac{-v_{c c}}{2 R_{1}}$ to $\sim \frac{v_{c c}}{2 R_{1}}$ and is not 


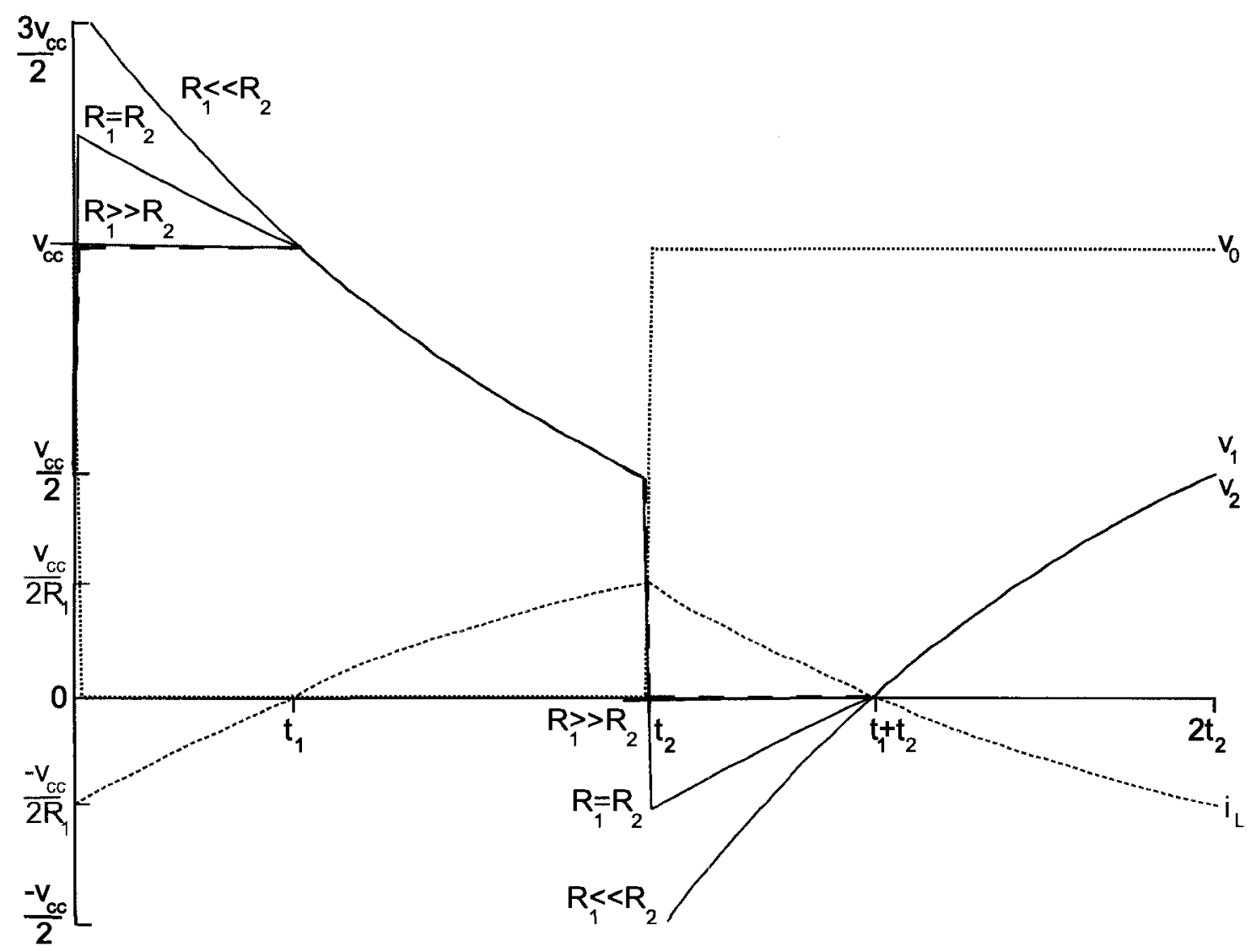

Figure 4.13: The timing diagram for the relaxation circuit. The current is magnified to a larger scale than the voltage. The frequency is higher when $R_{1}<<R_{2}$ and lower when $R_{1} \gg R_{2}$. 
to scale with the voltage. The three special cases of resistor choice are shown and it can be seen that when $R_{1}<<R_{2}$, the period decreases even though the voltage varies more.

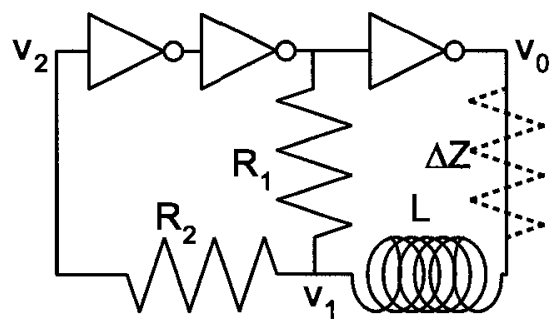

Figure 4.14: The effect of the conductivity change is modeled as a series resistor

Just as we did with the Colpitts circuit, we will now derive the expected frequency when an impedance $\Delta Z$ is added in series with the coil as shown in Fig. 4.14. The voltage at $v_{1}$ with the added resistance is calculated again through circuit analysis:

$$
\begin{aligned}
v_{1} & =\frac{v_{c c}\left[\Delta Z+R_{1} \| R_{2}\left(\frac{\Delta Z}{2 R_{1}}+\frac{2 R_{1}+3 R_{2}}{2 R_{1}+2 R_{2}}\right) e^{\left.\frac{-t\left(\Delta Z+R_{1} \| R_{2}\right)}{L}\right]}\right.}{\Delta Z+R_{1} \| R_{2}} \text { for } 0 \leq t \leq t_{1} \\
& =\frac{v_{c c}\left(\Delta Z+R_{1} e^{\frac{-\left(t-t_{1}\right)\left(\Delta Z+R_{1}\right)}{L}}\right)}{\Delta Z+R_{1}} \text { for } t_{1} \leq t \leq t_{2} \\
& =\frac{v_{c c}}{2} \text { for } t=t_{2} .
\end{aligned}
$$

The time intervals are recalculated,

$$
\begin{aligned}
& t_{2}=\frac{L \ln \left(\frac{2 R_{1}}{R_{1}-\Delta Z}\right)}{R_{1}+\Delta Z}+t_{1} \\
& t_{1}=\frac{L \ln \left(\frac{\Delta Z}{2 R_{1}}+\frac{2 R_{1}+3 R_{2}}{2 R_{1}+2 R_{2}}\right)}{R_{1} \| R_{2}+\Delta Z}
\end{aligned}
$$


and the frequency is then

$$
\begin{aligned}
f & =\frac{1}{2 t_{2}} \\
& =\frac{1}{2 L\left[\frac{1}{R_{1}+\Delta Z} \ln \left(\frac{2 R_{1}}{R_{1}-\Delta Z}\right)+\frac{1}{R_{1} \| R_{2}+\Delta Z} \ln \left(\frac{\Delta Z}{2 R_{1}}+\frac{2 R_{1}+3 R_{2}}{2 R_{1}+2 R_{2}}\right)\right]} .
\end{aligned}
$$

Once again, for the three special cases, the frequency calculation simplifies. If $R_{1}>>$ $R_{2}$, then $R_{1} \| R_{2} \simeq R_{2}$, and $\frac{2 R_{1}+3 R_{2}}{2 R_{1}+2 R_{2}} \simeq 1$, and so,

$$
\begin{aligned}
f & \simeq \frac{1}{2 L\left[\frac{1}{R_{1}+\Delta Z} \ln 2+\frac{1}{R_{2}+\Delta Z} \ln \left(1+\frac{\Delta Z}{R_{1}}\right)\right]} \\
& \simeq \frac{R_{1}+\Delta Z}{2 L \ln 3.3}, \text { if } R_{1} \gg R_{2} .
\end{aligned}
$$

If $R_{1}<<R_{2}$ then $R_{1} \| R_{2} \simeq R_{1}$, and $\frac{2 R_{1}+3 R_{2}}{2 R_{1}+2 R_{2}} \simeq \frac{3}{2}$, so,

$$
\begin{aligned}
f & \simeq \frac{R_{1}+\Delta Z}{2 L \ln \left(\frac{3 R_{1}+\Delta Z}{R_{1}-\Delta Z}\right)} \\
& \simeq \frac{R_{1}+\Delta Z}{2 L \ln 3}, \text { if } R_{1} \ll R_{2} .
\end{aligned}
$$

If $R_{1}=R_{2}$ then $R_{1} \| R_{2}=\frac{R_{1}}{2}$, and $\frac{2 R_{1}+3 R_{2}}{2 R_{1}+2 R_{2}}=\frac{5}{4}$. We rearrange the frequency calculation and make some simplifications to find that:

$$
\begin{aligned}
f & =\frac{R_{1}+\Delta Z}{2 L \ln \left[\left(\frac{2 R_{1}}{R_{1}-\Delta Z}\right)\left(\frac{\Delta Z}{2 R_{1}}+\frac{5}{4}\right)^{\left(\frac{2 R_{1}+2 \Delta Z}{R_{1}+2 \Delta Z}\right)}\right.} \\
& \simeq \frac{R_{1}+\Delta Z}{2 L \ln \left(\frac{\Delta Z^{2}}{2 R_{1}^{2}}+\frac{5 \Delta Z}{2 R_{1}}+\frac{25}{8}\right)} \\
& \simeq \frac{R_{1}+\Delta Z}{2 L \ln \left(\frac{25}{8}\right)}, \text { if } R_{1}=R_{2} .
\end{aligned}
$$


However, the frequency follows more closely to a corrected model,

$$
f \simeq \frac{R_{1}+\Delta Z}{2 L \ln \left(\frac{4 \Delta Z}{R_{1}}+\frac{25}{8}\right)}, \text { if } R_{1}=R_{2},
$$

where constants were determined heuristically.

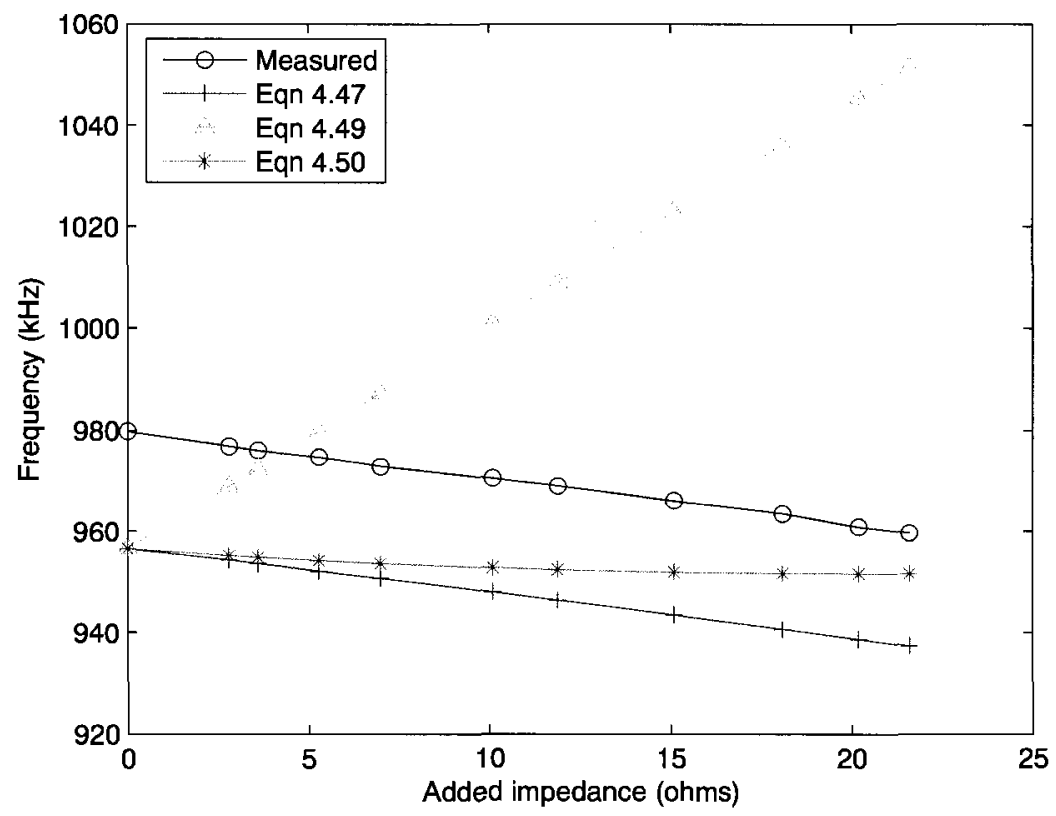

Figure 4.15: Small series resistors were added to the relaxation circuit and the frequency was measured

We tested this effect by adding resistors in series with the inductor in the relaxation circuit and measuring the frequency. The circuit components used were $R_{1}=R_{2}=218 \Omega$, and $L=100 \mu \mathrm{H}$ and the results are shown in Fig. 4.15. The offset between measured and theoretical frequencies is likely due to the inductor being an unideal component and capacitive coupling as the circuit was built on a breadboard. The theoretical frequency (Eqn. 4.47) follows the measured frequency very closely and Eqn. 4.50 is also a reasonable estimate, whereas Eqn. 4.49 gives quite different predicted frequencies. Note however, that the simplifications we used for Eqn. 4.49 were 
based on the assumption that $\Delta Z<<R_{1}$. The added resistors in this experiment are large relative to $R_{1}$ and $R_{2}$ compared to what we would expect from an induced impedance, so our simplifications to the theoretical frequency are still reasonable for our sensor.

We can now calculate our relative change in frequency parameter, and we find that all three instances of resistor selection result in the same conclusion:

$$
\frac{\Delta f}{f} \simeq \frac{\Delta Z}{R_{1}}
$$

\subsubsection{Circuit Optimization}

From Eqn. 4.51 it would appear that we should maximize $\Delta Z$ and minimize $R_{1}$. However, in our derivation of induced resistance in the last chapter, we showed that $\Delta Z \propto \omega^{2}$ and we know that the frequency of the relaxation circuit is proportional to $R_{1}$. Substituting Eqn. 3.30, the proportionality formula for $\Delta Z$, and Eqn. 4.22, the theoretical inductance of an air core coil into Eqn. 4.51,

$$
\begin{aligned}
\frac{\Delta f}{f} & \propto \frac{\omega^{2} N^{2} \Delta \sigma F_{\text {coil }}(R)}{R_{1}} \\
& \propto \frac{R_{1} N^{2} \Delta \sigma F_{\text {coil }}(R)}{L^{2}} \\
& \propto \frac{(6 R+9 l+10 d)^{2} R_{1} \Delta \sigma F_{\text {coil }}(R)}{R^{4} N^{2}} .
\end{aligned}
$$

where we have made the substitution $\omega \propto \frac{R_{1}}{L}$. Thus, we once again want to maximize the coil length $l$ and coil thickness $d$. If we only make our coil one layer thick, then the coil thickness is simply the wire diameter and the coil length is the product of the wire diameter and the number of turns. We will assume our wire diameter is about 
$0.5 \mathrm{~mm}$ and our expression then becomes,

$$
\frac{\Delta f}{f} \propto \frac{(6 R+0.0045 N+0.005)^{2} R_{1} \Delta \sigma F_{\text {coil }}(R)}{R^{4} N^{2}}
$$

It is clear from this expression that we want to maximize the resistor $R_{1}$ but the optimal coil radius and number of turns are less obvious. We determine through simulations that they should both be minimized.

Referring to Eqns. 4.33-4.35, the frequency also increases slightly as $R_{1}$ decreases relative to $R_{2}$, but there is only about eight percent difference between the two extremes. According to the derived equations, the choice of $R_{1}$ has a much greater effect on the operating frequency and consequently the induced impedance than does the choice of $R_{2}$. We tested this by measuring the frequency of the circuit with different values of $R_{1}, R_{2}$, and $L$, and the results matched the theoretical predictions.

\subsection{Inductive Coil}

It is clear from Eqns. 4.25 and 4.54 that the coil properties are an important factor in optimizing the circuits. Several inductive coils were handmade and tested. The coils were made from thin insulated wire taken from old disk drives. Water bottles were wrapped in parchment paper to act as cylindrical molds. The wire was wound tightly in a single layer around the mold, taped in place, and then superglued together. Once the glue had dried, the water bottle was slipped out and the tape and parchment paper were removed, leaving behind a cylindrical coil. Coils were made with different radii and numbers of turns. The coil thickness was given by the diameter of the wire and the coil length was dependent on the number of turns.

The inductance of the coils was measured with a Hewlett-Packard 4263B LCR 


\begin{tabular}{|l|l|l|l|l|l|}
\hline $\mathbf{R}(\mathbf{c m})$ & $\mathbf{N}$ & $\mathbf{l}(\mathbf{c m})$ & $\mathbf{L}_{\mathbf{t}}(\mu \mathbf{H})$ & $\mathbf{L}_{\mathbf{m}} @ \mathbf{1 0 0 H z}(\mu \mathbf{H})$ & $\mathbf{L}_{\mathbf{m}} @ \mathbf{1 0 0 k H z}(\mu \mathbf{H})$ \\
\hline \multirow{2}{*}{3.7} & 10 & 0.4 & 16.4 & 18.4 & 18.0 \\
& 15 & 0.5 & 35.7 & 36.8 & 36.4 \\
\hline \multirow{2}{*}{4} & 10 & 0.4 & 17.9 & 21.3 & 20.2 \\
& 15 & 0.5 & 39.1 & 41.6 & 40.8 \\
\hline \multirow{2}{*}{4.6} & 10 & 0.3 & 21.6 & 20.5 & 20.1 \\
& 15 & 0.5 & 46.0 & 43.2 & 42.9 \\
\hline
\end{tabular}

Table 4.1: Properties of the coils for the in-vivo trials. $R$ is the coil radius, $N$ is the number of loops in the coil, $l$ is the coil length, $L_{t}$ is the theoretical coil inductance, $L_{m} @ 100 H z$ is the coil inductance measured at $100 \mathrm{~Hz}$, and $L_{m} @ 100 k H z$ is the coil inductance measured at $100 \mathrm{kHz}$

\begin{tabular}{|c|c|c|c|}
\hline $\mathbf{R}(\mathbf{c m})$ & $\mathbf{N}$ & $\mathbf{L}_{\mathbf{m}} @ \mathbf{1 0 0 k H z}(\mu \mathbf{H})$ & $\mathbf{Q}$ \\
\hline 4.15 & 8 & $14.9 \pm 0.1$ & $9.7 \pm 0.1$ \\
\hline 4.15 & 10 & $17.75 \pm 0.01$ & $14.5 \pm 0.1$ \\
\hline 4.15 & 12 & $29.31 \pm 0.01$ & $19.4 \pm 0.1$ \\
\hline
\end{tabular}

Table 4.2: The properties of coils made for some phantom trials. $R$ is the coil radius, $N$ is the number of loops in the coil, $L_{m} @ 100 k H z$ is the coil inductance measured at $100 \mathrm{kHz}$, and $\mathrm{Q}$ is the measured quality factor.

Meter and Hewlett-Packard 16089B Kelvin Clip leads. Table 4.1 lists the theoretical and measured inductances of the coils with the different coil parameters. The theoretical inductance is given by Eqn. 4.22 .

Also, for one set of phantom trials, three coils were each made by winding the wire tightly in a single layer around a graduated cylinder and taping it in place. The coil remained taped to the cylinder for the duration of the trial and was removed afterward. The outer diameter of the cylinder was $8.3 \mathrm{~cm}$ and the coils were made with 8,10 , and 12 turns. The inductance and quality factor were again measured with the same LCR meter and leads and the measurement frequency was $100 \mathrm{kHz}$. Table 4.2 shows the properties of the coils that were used for this set of phantom trials.

Since we wanted to use the same coil for both circuits in order to compare their 
sensitivities, we had to compromise between the optimal circuit scenarios for each and thus the 10 turn coil with radius $4.6 \mathrm{~cm}$ was chosen for the in vivo trials.

\subsection{Component Selection}

All circuits were built on breadboards and tested using standard laboratory equipment. Several circuits were also soldered to prototype boards and tested. Once the final circuit designs and components were decided, the two circuits were soldered to prototype boards and these boards were used for the phantom and in vivo experiments.

The tank capacitors of the Colpitts oscillator were chosen as $C_{1}=C_{2}=390 \mathrm{pF}$. Small tank capacitors were chosen in order to increase the frequency of oscillation. The value of $R_{2}$ was decreased as far as possible while still maintaining oscillations in order to lower the gain and make the circuit marginally stable and thus more reactive to small changes in coil properties. The resistor values chosen were $R_{2}=56$ $\mathrm{k} \Omega$ and $R_{1}=R_{3}=R_{4}=10 \mathrm{k} \Omega$. The other capacitor values were $C_{3}=10 \mathrm{pF}$ and $C_{4}=C_{5}=C_{6}=100 \mathrm{nF}$. The resistors had a $5 \%$ tolerance so the components were measured for accuracy using a Wavetek DM15XL multimeter for resistances and the HP LCR meter and Kelvin Clip leads set at $100 \mathrm{kHz}$ for the capacitances. The measurements were as follows: $R_{1}=10.10 \pm 0.01 \mathrm{k} \Omega, R_{2}=55.8 \pm 0.1 \mathrm{k} \Omega$, $R_{3}=9.81 \pm 0.01 \mathrm{k} \Omega, R_{4}=9.82 \pm 0.01 \mathrm{k} \Omega, C_{1}=401 \pm 1 \mathrm{pF}, C_{2}=408 \pm 1 \mathrm{pF}$, $C_{3}=12 \pm 1 \mathrm{pF}, C_{4}=103 \pm 0.1 \mathrm{nF}, C_{5}=104 \pm 0.1$ and $C_{6}=98 \pm 0.1 \mathrm{nF}$.

The final version of the relaxation circuit was built with $R_{1}=R_{2}=330 \Omega \pm 5 \%$ and a $100 \mathrm{nF}$ capacitor bridging the power supply. The measured values of the components were $R_{1}=R_{2}=326 \pm 1 \Omega$, and $C=107.15 \pm 0.1 \mathrm{nF}$. 


\subsection{Frequency Measurement}

The change in frequency that the oscillator experiences is very small relative to the operating frequency. During the preliminary investigation, we also built a test circuit that produced a down converted signal which would be more sensitive to small changes in frequency. Basically, we were subtracting a base frequency from our signal in order to increase the relative change in frequency.

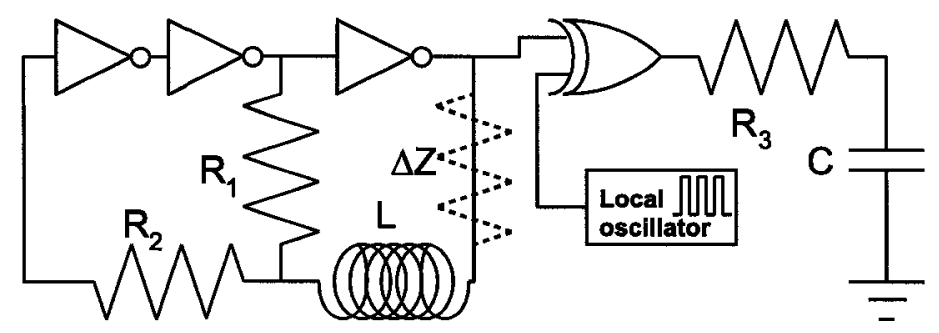

Figure 4.16: A test circuit with the relaxation oscillator fed through a mixer and filter

The output from the relaxation circuit and the output from a frequency generator were fed into an "exclusive or" gate and then fed through an RC low pass filter as seen in Fig. 4.16. The advantage of this approach is that the output is a continuous signal in time that is directly proportional to the change in frequency which means that the output signal could then be amplified and it could also be easily compared to a signal measuring the change in conductivity or a measurement of a participants breathing with time. Unfortunately, in our investigation we found that the two input signals had a tendency of locking into the same frequency whenever their difference was small, which was precisely the scenario we were most interested in. We concluded that this method was not reliable, and the oscillator frequency was measured with a spectrum analyzer instead. 


\subsection{Summary}

This chapter presented the two electronic circuits that we studied and tested. The resonance frequencies were derived for both circuits as well as the relative changes in frequency we expect from a nearby conductive volume, which we modeled as an impedance $\Delta Z$ in series with the inductor. We presented a method for choosing circuit components to optimize the sensitivity of the coil and we listed the final component selections and coil properties. 


\section{Chapter 5}

\section{Experiments}

Experiments were conducted with phantoms as well as with human participants. Phantom trials were done to see whether or not changes to a conductive volume would be detectable with the circuits and to test the validity of the theoretical model derived in Chapter 3. In vivo trials were then conducted with participants to evaluate the feasibility of this technique for detecting changes in lung volume.

This chapter chronicles all of the experimental trials. The experimental setup, including the equipment that was used, is described and the results of each experiment are provided. Finally, the results and sources of error are discussed.

\subsection{Phantom Trials}

The goal of the phantom trials was to compare experimental data to the theoretical model we derived. The phantom trials provide known geometries and values that can be matched to the theoretical model, whereas the in-vivo experiments involve multiple assumptions and estimates. In order to simulate our theoretical model of a person breathing as close as possible, several attempts were made to design a setup with 
a spherical phantom with an adjustable conductivity and height above the circuit. These setups proved too cumbersome and required too much user interference. Since we were attempting to measure very small changes in frequency from changes in the conductivity of the surroundings, any alterations made by the user to the setup would have potentially created enough noise to overwhelm our signal. Consequently, a simple cylinder phantom was adopted instead.

\subsubsection{Experimental Setup}

A plastic $2000 \mathrm{~mL}$ graduated cylinder, filled with conductive liquid, was used to make cylindrical phantoms of known conductivity and size. The cylinder had an outer diameter of about $8.3 \mathrm{~cm}$ and inner diameter of about $7.9 \mathrm{~cm}$, but the diameter varied slightly from top to bottom. The volume of the liquid and the positioning of the inductive coil were adjusted to make changes to the phantom height values. The liquid was made by mixing tap water with sodium chloride in varying proportions to set the conductivity.

A hand-held ECTestr High device by Eutech Instruments and Oakton Instruments was used to measure the conductivity of the liquid used in the phantom trials. A second device, Fisher Scientific RC-16B2 Conductivity Bridge by Industrial Instruments (Cedar Grove, N.J.) was used to verify the conductivity measurements. The cell constant of the conductivity bridge was measured as $19.4 \pm 0.8$ using $9.7 \mu \mathrm{S} / \mathrm{cm}$ Traceable Conductivity Calibration Standard by Control Company (Friendswood, Texas). The conductivity of the calibration fluid was very low and therefore on a different scale of the conductivity bridge than salt water measurements and comparison with the ECTestr readings gave a cell constant estimate of closer to 23 . We concluded that the measurements from the conductivity bridge concurred with those of the ECTestr.

A Tektronix TDS 3014 Oscilloscope was used to monitor the circuit and determine 
the approximate resonance frequency. A Hewlett-Packard 3585A Spectrum Analyzer was used to measure the frequency of the circuit during each trial by manually adjusting the display and observing the peak frequency. The circuit was connected to the spectrum analyzer inductively due to an impedance mismatch.

Trials were conducted with different coils either at the base of the cylinder or wrapped around the cylinder at the $500 \mathrm{~mL}$ mark.

\subsubsection{Phantom Trial \#1}

The first trial investigated the effect that the conductivity and height of the phantom had on the change in frequency. Preliminary trials were done in the following manner with up to $1000 \mathrm{~mL}$ of liquid of conductivity 0.01 to $0.1 \mathrm{~S} / \mathrm{m}$. The conductivity of the liquid was measured using both the ECTestr and the conductivity bridge. The 10 turn coil with a $4.6 \mathrm{~cm}$ radius was placed around the cylinder at its base. The liquid was then added to the cylinder in increments of $200 \mathrm{~mL}$ and the frequency of the circuit was measured using the oscilloscope and spectrum analyzer. This procedure was repeated for the second circuit and then the conductivity of the liquid was increased for the next set of measurements. We did not get significant changes in frequency from either circuit from these trials, but we were able to show that the ECTestr was sufficient for measuring the conductivity of the liquid and we determined that the liquid needed higher conductivity for conclusive frequency change results.

The previous tests were repeated using liquid conductivities of $0.01,0.61,0.98$, 1.33 , and $1.88 \pm 0.01 \mathrm{~S} / \mathrm{m}$ for the Colpitts circuit and $0.01,0.59,0.93,1.31$, and $1.85 \pm$ $0.01 \mathrm{~S} / \mathrm{m}$ for the relaxation circuit. The procedure was changed to doing all the tests on one circuit and then doing them all on the second circuit so that the circuits would not have to be constantly switched and reconnected. It was hoped that this would make the base frequency and circuit behaviour more consistent. We observed, 


\begin{tabular}{|l||c||c|c|c|c|c|}
\hline \multicolumn{1}{|l|}{} & \multicolumn{1}{c||}{$\begin{array}{c}\text { Conductivity } \\
\pm 0.01 \mathrm{~S} / \mathrm{m}\end{array}$} & $f_{0} \pm 200 \mathrm{~Hz}$ & \multicolumn{5}{c|}{$\Delta f=f-f_{0} \pm 400 \mathrm{~Hz}$} \\
\cline { 2 - 7 } & $0 \mathrm{~mL}$ & $200 \mathrm{~mL}$ & $400 \mathrm{~mL}$ & $600 \mathrm{~mL}$ & $800 \mathrm{~mL}$ & $1000 \mathrm{~mL}$ \\
\hline 0.01 & 2448400 & 0 & -200 & -200 & -200 & -200 \\
\hline 0.61 & 2448000 & -200 & -400 & -400 & -600 & -400 \\
\hline 0.98 & 2448800 & -200 & -200 & -200 & -400 & -200 \\
\hline 1.33 & 2448600 & 0 & 0 & 0 & -200 & -200 \\
\hline 1.88 & 2449000 & 0 & 0 & 0 & 0 & 0 \\
\hline
\end{tabular}

Table 5.1: Trial \#1 Colpitts circuit results. The starting frequency $f_{0}$ and change in frequency $\Delta f$ with different phantom conductivities and heights using the Colpitts circuit and a 10 turn coil with $4.6 \mathrm{~cm}$ radius at the base of the cylinder.

however, that the base frequency was still not constant as the coil was moved each time the cylinder was emptied. A lid was placed over the circuit as a precaution in order to prevent air currents and reduce temperature changes in the circuit which would affect the frequency. The power to the circuit was left on for a while to let the circuit warm up before each set of measurements and all other equipment was left on the entire time. The span on the spectrum analyzer was set to $200 \mathrm{kHz}$ and the measurement error was $\pm 200 \mathrm{~Hz}$ for both circuits.

The results are presented in Tables 5.1 and 5.2 for the Colpitts and relaxation circuits. The average starting frequencies, meaning the frequency when the cylinder was empty, were $2448560 \pm 200 \mathrm{~Hz}$ for the Colpitts circuit and $4922040 \pm 200 \mathrm{~Hz}$ for the relaxation circuit and the theoretical resonance frequencies are $2.52 \mathrm{MHz}$ and 7.06 $\mathrm{MHz}$ respectively.

The measured relative change in frequency parameters $\frac{\Delta f}{f}$ along with the theoretical values predicted using our model are plotted in Fig. 5.1 and Fig. 5.2 for the Colpitts and relaxation circuits respectively. The theoretical relaxation circuit predictions are displayed at ten times the scale of the measured results in Fig. 5.2. Errors were estimated from measurement errors. We use $f_{0}$ from Tables 5.1 and 5.2 for calculations of both our measured and theoretical change in frequency parameters. We 


\begin{tabular}{|l||c||c|c|c|c|c|}
\hline \multicolumn{1}{|l|}{$\begin{array}{l}\text { Conductivity } \\
\pm 0.01 \mathrm{~S} / \mathrm{m}\end{array}$} & $f_{0} \pm 200 \mathrm{~Hz}$ & \multicolumn{5}{c|}{$\Delta f=f-f_{0} \pm 400 \mathrm{~Hz}$} \\
\cline { 2 - 7 } & $0 \mathrm{~mL}$ & $200 \mathrm{~mL}$ & $400 \mathrm{~mL}$ & $600 \mathrm{~mL}$ & $800 \mathrm{~mL}$ & $1000 \mathrm{~mL}$ \\
\hline 0.01 & 4926000 & -1000 & -1400 & -1800 & -2000 & -2400 \\
\hline 0.59 & 4921800 & -800 & -1200 & -1600 & -1800 & -2000 \\
\hline 0.93 & 4921000 & -200 & -400 & -1200 & -1600 & -1800 \\
\hline 1.31 & 4919800 & 0 & -600 & -1000 & -1200 & -1400 \\
\hline 1.85 & 4921600 & 200 & -200 & -1200 & -1400 & -1400 \\
\hline
\end{tabular}

Table 5.2: Trial \#1 relaxation circuit results. The starting frequency $f_{0}$ and change in frequency $\Delta f$ with different phantom conductivities and heights using the relaxation circuit and a 10 turn coil with $4.6 \mathrm{~cm}$ radius at the base of the cylinder.

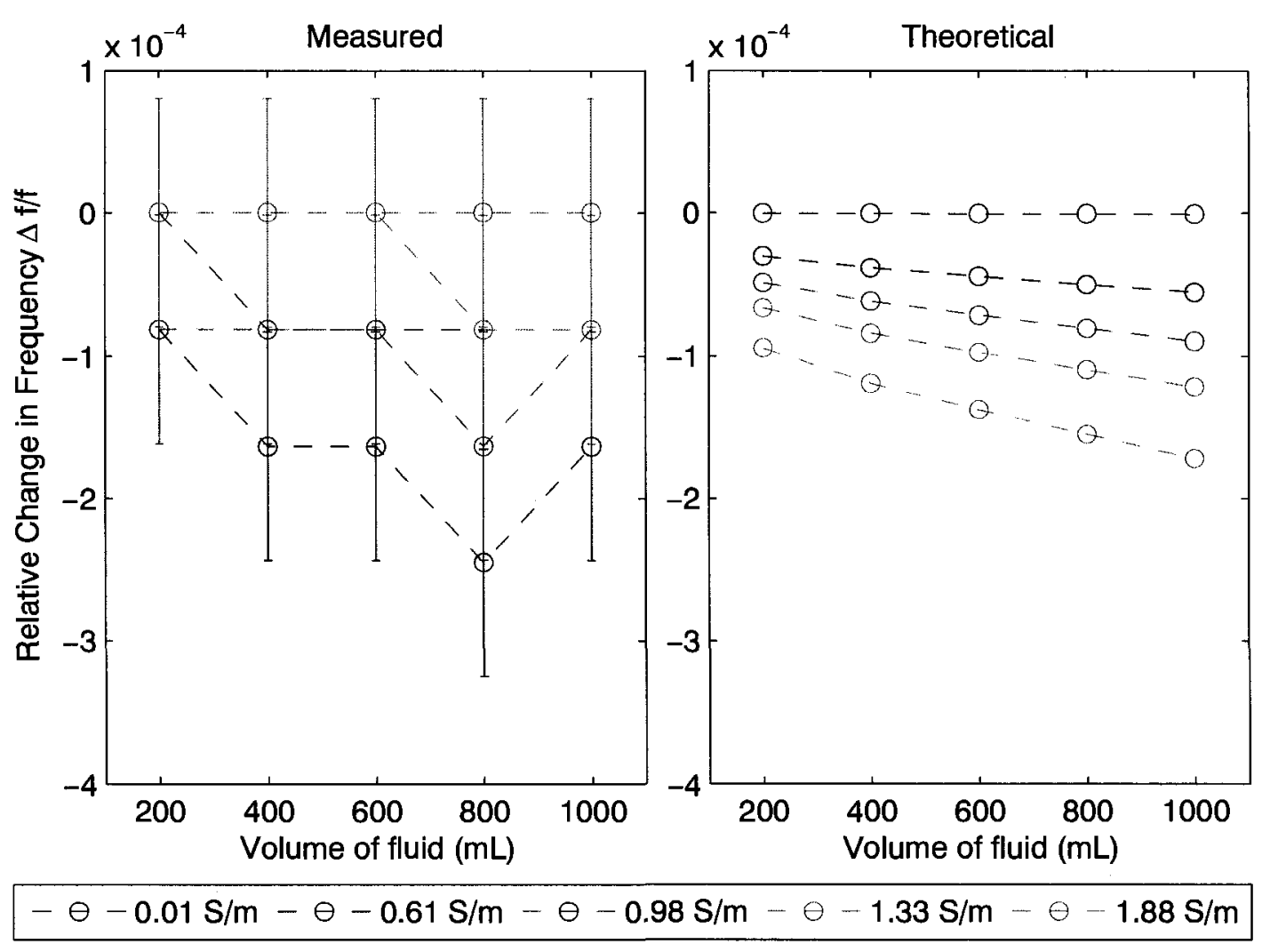

Figure 5.1: The relative changes in frequency measured and theoretically predicted for phantom trial \#1 with the Colpitts circuit 

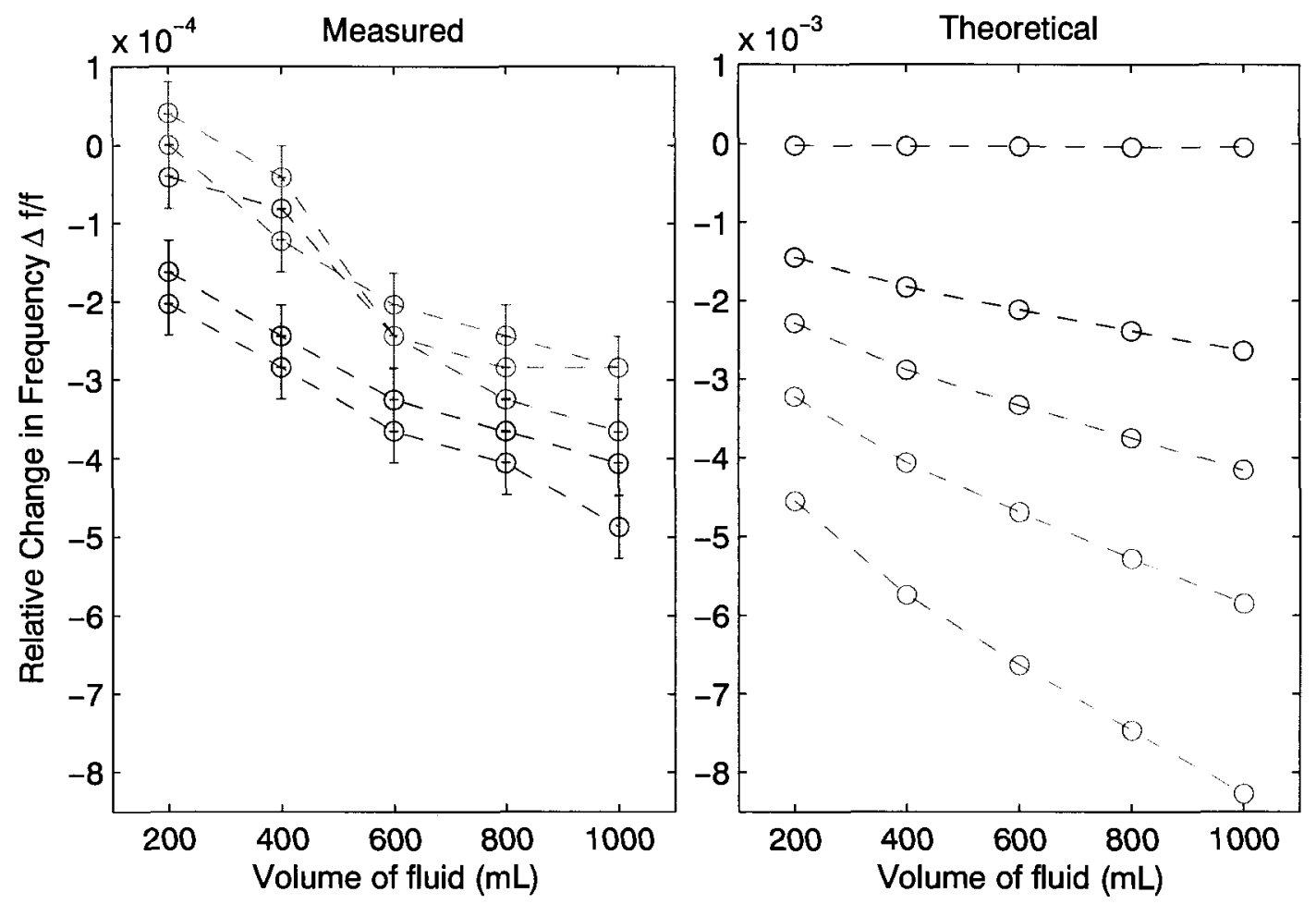

$-\ominus-0.01 \mathrm{~S} / \mathrm{m}-\ominus-0.59 \mathrm{~S} / \mathrm{m}-\ominus-0.93 \mathrm{~S} / \mathrm{m}-\ominus-1.31 \mathrm{~S} / \mathrm{m}-\ominus-1.85 \mathrm{~S} / \mathrm{m}$

Figure 5.2: The relative changes in frequency measured and theoretically predicted for phantom trial \#1 with the relaxation circuit. 
say the radius of the conductive volume is $3.95 \mathrm{~cm}$ and the height is $4.08 \mathrm{~cm}$ for each $200 \mathrm{~mL}$ increment.

Our theoretical model predicted an induced impedance proportional to the conductivity of the phantom, but the experimental measurements do not fully support this theory. We expected to see an increase in the magnitude of the frequency change as the conductivity increases and as the height of the phantom increases (though this drops off quickly with the distance from the coil). The measured relative frequency changes do not correlate very well with the theoretical prediction and we conclude that the span on the spectrum analyzer should have been set smaller to make more accurate measurements for this trial, particularly with the Colpitts circuit.

\subsubsection{Phantom Trial \#2}

The purpose of the second trial was to test how the sensor was affected by changes to the number of loops $N$ of the coil, the distance $z_{0}$ between the coil and the phantom, and the height $h_{0}$ of the phantom. This trial used salt water with a conductivity that exceeded the range of the ECTestr device. The conductivity bridge indicated that the liquid had a conductivity of $0.165 \pm 0.005 *$ cellconstant $=3.8 \pm 0.3 \mathrm{~S} / \mathrm{m}$ where we have taken the cell constant to be $23 \pm 1$. For this trial, coils with 8,10 , and 12 turns were taped to the $500 \mathrm{~mL}$ mark as described in the last chapter. The coil properties are listed in Table 4.2 .

The frequency was measured at $200 \mathrm{~mL}$ increments up to $1000 \mathrm{~mL}$. The frequency was also measured at the $500 \mathrm{~mL}$ mark. The cylinder was then emptied and the procedure was repeated. Two sets of measurements were taken per circuit with the 10 turn coil and three sets per circuit were taken with the 8 and 10 turn coils. The frequency span of the analyzer was set to $10 \mathrm{kHz}$ for the Colpitts circuit measurements and $100 \mathrm{kHz}$ for the relaxation circuit measurements. The measurement error was 


\begin{tabular}{|l|l||c||c||c|}
\hline \multicolumn{2}{|c||}{} & $\mathrm{N}=8$ & $\mathrm{~N}=10$ & $\mathrm{~N}=12$ \\
\hline \multirow{2}{*}{ Colpitts } & $\bar{f}_{0}(\mathrm{~Hz})$ & $2672797 \pm 50$ & $2546625 \pm 50$ & $2040843 \pm 50$ \\
\cline { 2 - 5 } & $f_{t}(\mathrm{MHz})$ & 2.95 & 2.71 & 2.11 \\
\hline \multirow{2}{*}{ Relaxation } & $f_{0}(\mathrm{~Hz})$ & $4901433 \pm 200$ & $4998050 \pm 200$ & $3687700 \pm 200$ \\
\cline { 2 - 5 } & $f_{t}(\mathrm{MHz})$ & 9.72 & 8.16 & 4.94 \\
\hline
\end{tabular}

Table 5.3: Trial \#2 average starting frequency $\bar{f}_{0}$ and theoretical resonance frequency $f_{t}$ of both circuits using coils of $N$ turns and radius $4.15 \mathrm{~cm}$ taped to the cylinder at the $500 \mathrm{~mL}$ mark.

$\pm 50 \mathrm{~Hz}$ for the Colpitts circuit and $\pm 200 \mathrm{~Hz}$ for the relaxation circuit.

The average starting frequencies $\bar{f}_{0}$ and the theoretical resonant frequencies $f_{t}$ are listed in Table 5.3. The theoretical resonant frequencies are calculated using the measured inductance of the coils.

Fig. 5.3 shows the measured and theoretical relative changes in frequency parameter $\frac{\Delta f}{f}$ as a function of the volume added for each of the three coils with the Colpitts circuit. Fig. 5.4 shows the equivalent information for the relaxation coil but the theoretical predictions are displayed at ten times the scale of the measured results. Errors were estimated from measurement errors. The measured change in frequency parameter is calculated as follows:

$$
\frac{\Delta \bar{f}}{f}=\frac{\bar{f}-\bar{f}_{0}}{\bar{f}_{0}}
$$

and the theoretical change in frequency parameter was derived in Chapter 4. Note that the results with the 10 turn coil were averaged over two trials whereas those for the 8 and 12 turn coils were averaged over three trials.

The measured results show similar curves of relative frequency changes, but with smaller magnitudes compared to the theoretical model. 

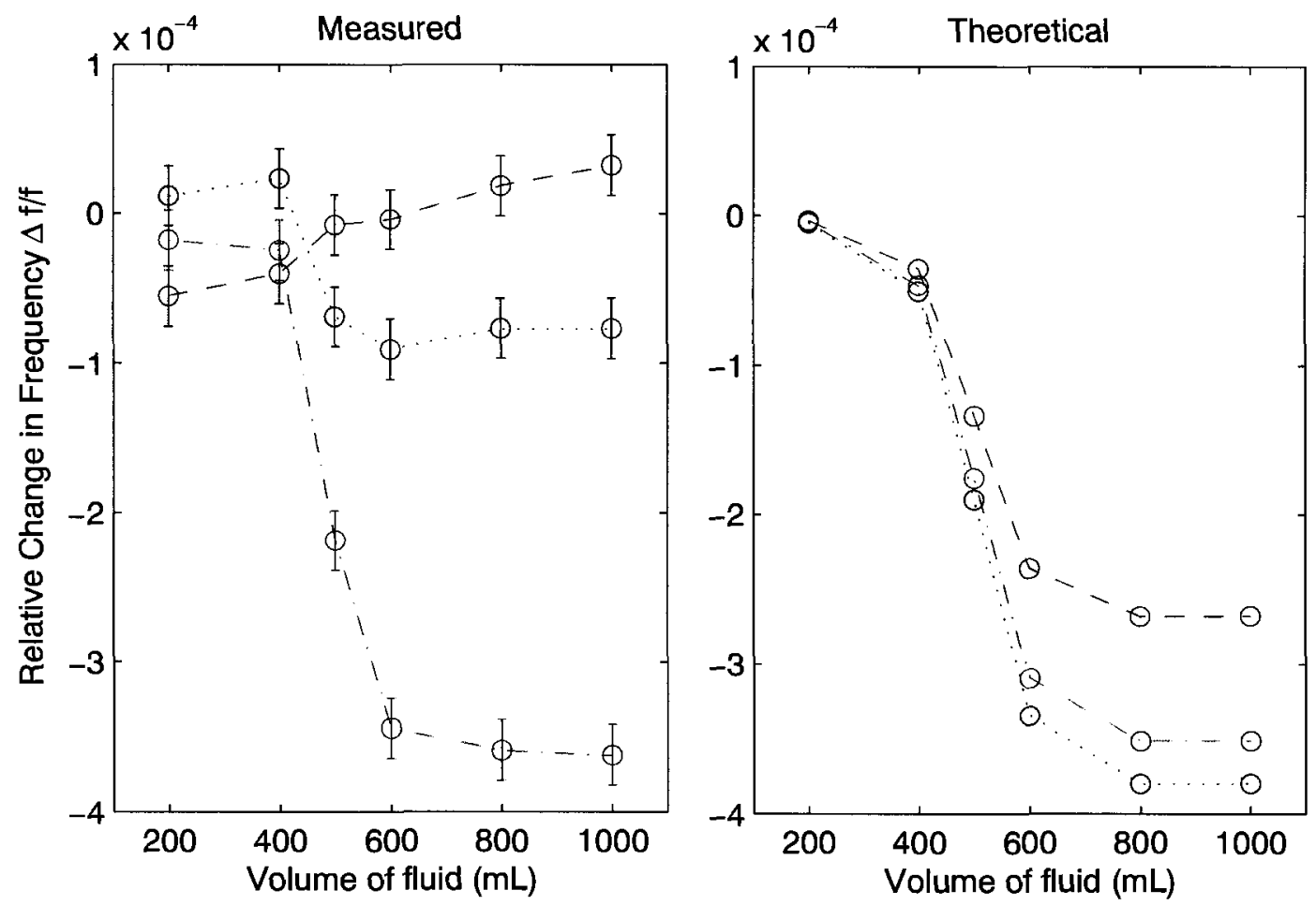

$$
-\ominus-N=8 \cdots \odot \cdots N=10 \quad-O-N=12
$$

Figure 5.3: The relative change in frequencies measured and theoretically predicted for phantom trial \#2 with the Colpitts circuit 

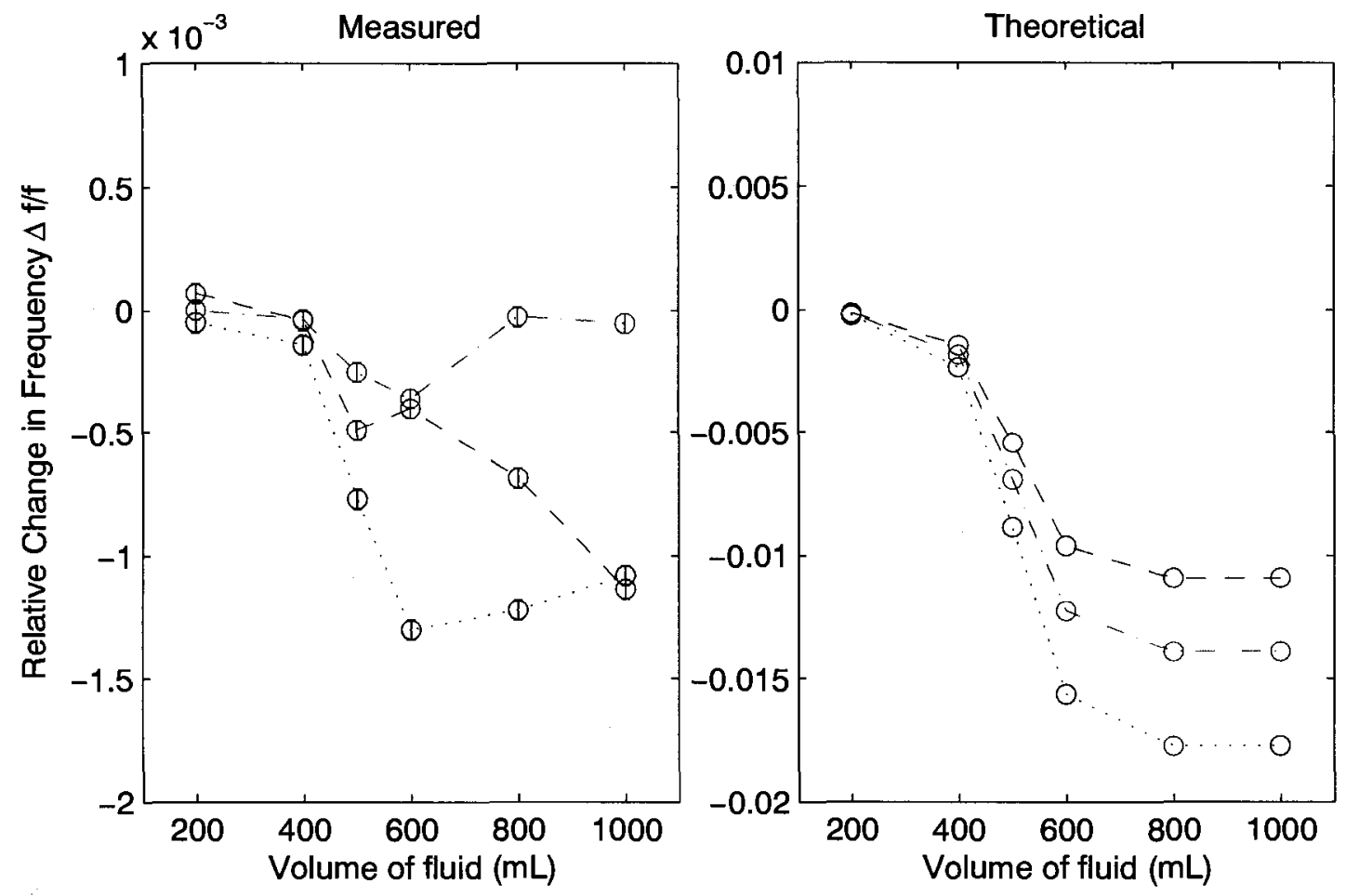

$$
-\ominus-N=8 \cdots \odot \cdots N=10 \quad-O-N=12
$$

Figure 5.4: The relative change in frequencies measured and theoretically predicted for phantom trial \#2 with the relaxation circuit 


\subsubsection{Phantom Trial \#3}

The goal of the final trial was to verify previous measurements and to specifically address the relationship between the conductivity of the liquid and the frequency change of the circuit. Results from a preliminary investigation in which highly conductive liquid was added to one litre of water suggested that the change in frequency was not as high as theoretically predicted, so the experiment was repeated in a more regimented fashion.

In this test, the 8 turn coil was taped to the cylinder at the $500 \mathrm{~mL}$ mark. Tap water was added in increments of $100 \mathrm{~mL}$ up to the $1000 \mathrm{~mL}$ mark and the frequency of the circuit was measured at each stage. Highly conductive salt water was then added to the litre of tap water in $50 \mathrm{~mL}$ increments up to the $1300 \mathrm{~mL}$ mark. At each stage the fluid was mixed to give it a uniform conductivity, then the frequency of the circuit and conductivity of the fluid were measured. This test was conducted for both the Colpitts and relaxation circuits. The frequency span of the spectrum analyzer was set to $10 \mathrm{kHz}$ for the Colpitts circuit and $100 \mathrm{kHz}$ for the relaxation circuit. The measurement error was $\pm 50 \mathrm{~Hz}$ for the Colpitts circuit and $\pm 200 \mathrm{~Hz}$ for the relaxation circuit.

Fig. 5.5 shows the results of the third trial with the measured and theoretical relative changes in frequency and the measured conductivity. Error bars were estimated based on measurement errors. The theoretical model predicts very little change in frequency for the first $1000 \mathrm{~mL}$ since the change in conductivity is so low. However, relatively large changes in frequency were measured indicating that there is possibly another factor influencing the measurements. 

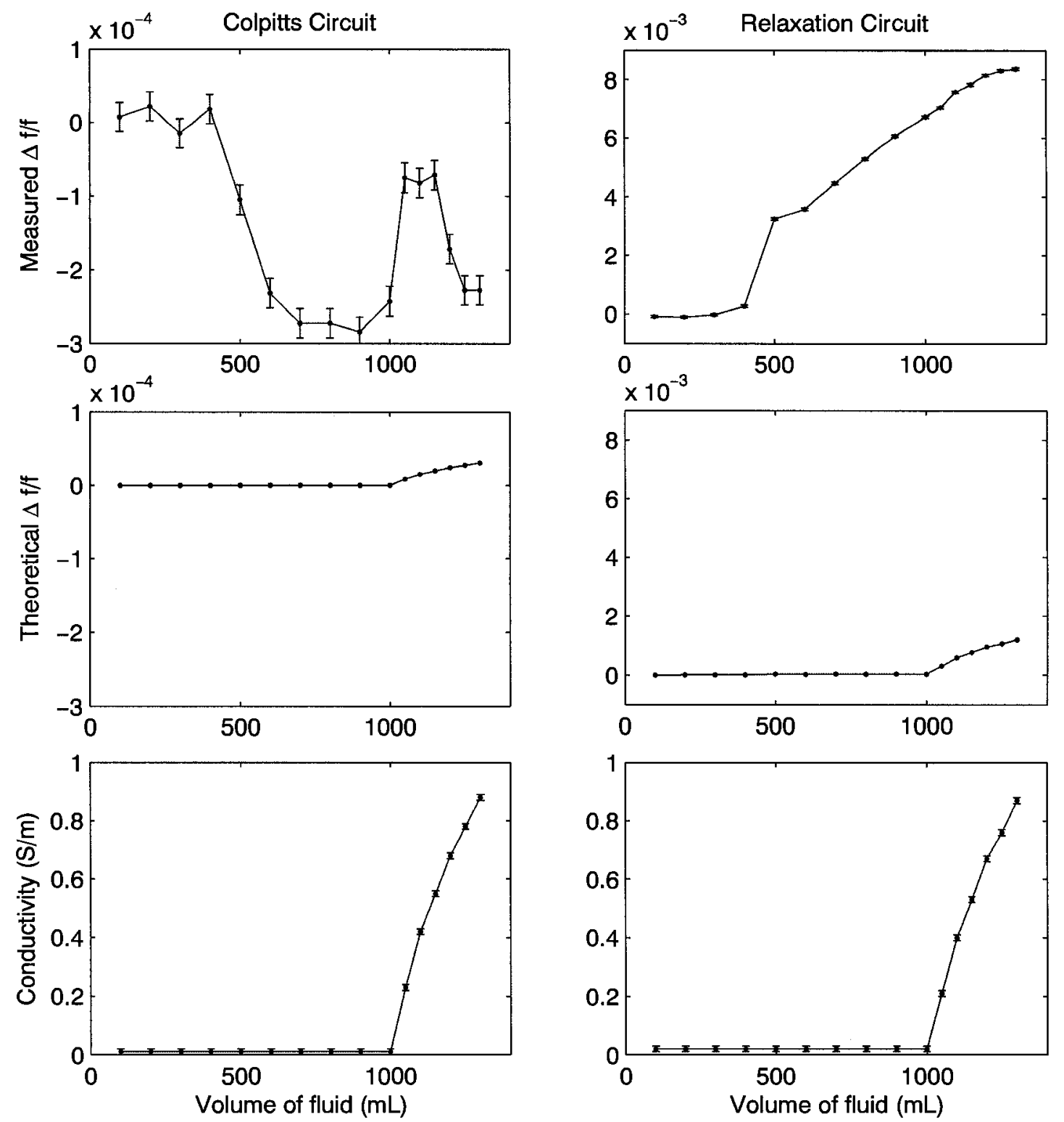

Figure 5.5: The relative change in frequencies measured and theoretically predicted for phantom trial \#3 as well as the conductivity measured for both the Colpitts and relaxation circuits 


\subsection{In Vivo Trials}

The in vivo trials were given ethical clearance by the Carleton University Research Ethics Committee. Six healthy participants (three males and three females) between the ages of 20 and 35 were recruited. The participants were given a letter of information outlining the purpose, procedure, risks and benefits, security and anonymity of data, withdrawal procedures, access to research findings, and contact information of the researchers and ethics committee representative. A copy of the Letter of Information and Consent is given in Appendix B.

The Colpitts op-amp circuit and the relaxation circuit were both used in the in vivo trials in order to compare the performance of the two. The 10 turn coil with a radius of $4.6 \mathrm{~cm}$ was used. There were three breathing manoeuvers involved in the trials, each with breath holds: maximal breathing, breathing to $1 \mathrm{~L}$, and breathing to $400 \mathrm{~mL}$. Practice trials were done to test the feasibility of the setup.

\subsubsection{Experimental Setup}

The experimental setup for the in vivo trials is shown in Fig. 5.6. Each participant lay supine on a table with the coil positioned under his or her right lung. Participants were asked to remove excess clothing such as sweaters and metal objects like belts, watches, and rings. The coil was housed in a plastic lid shown in Fig. 5.7 and 5.8 to protect it from deformation as the participant breathed. The circuit was covered with a bag of liquid at room temperature in order to help regulate the temperature of the circuit and protect it from air currents or movement of the leads.

The circuit was powered with a Hewlett-Packard E3630A Triple output DC Power Supply set to within 0.1 volts accuracy. A Tektronix $221360 \mathrm{MHz}$ Oscilloscope was used to verify that the circuit was operating properly. The frequency of the circuit 


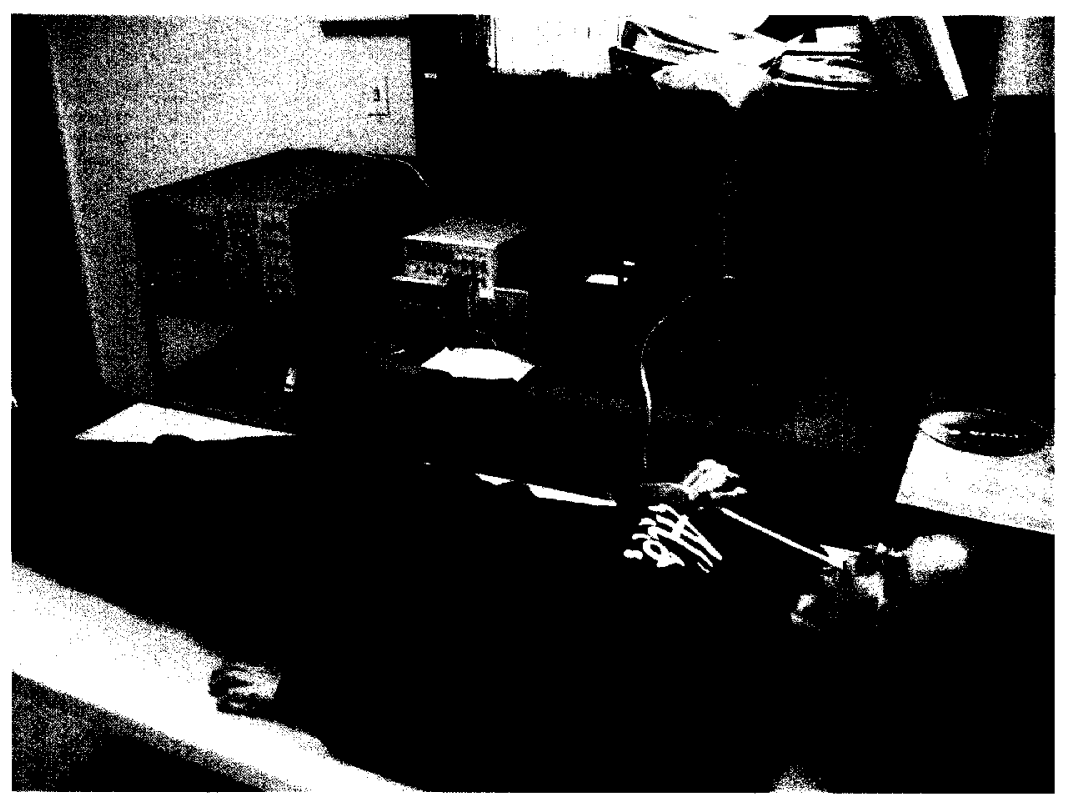

Figure 5.6: The experimental setup for the in vivo trials

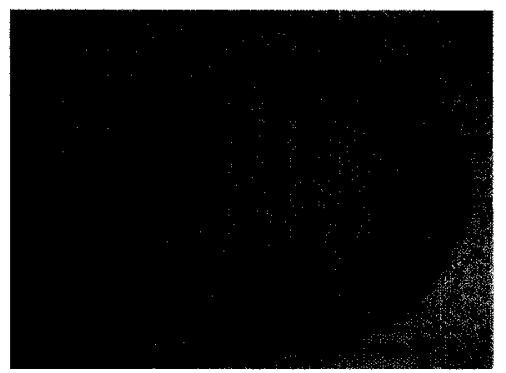

Figure 5.7: Coil with plastic lid covering it

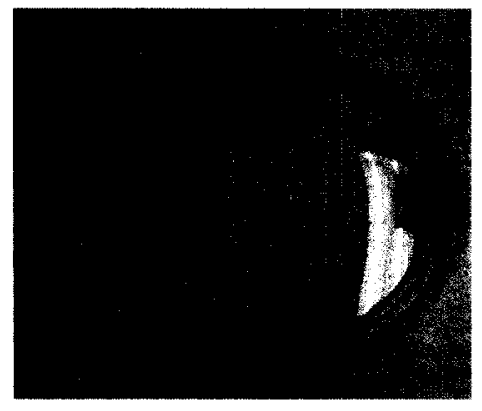

Figure 5.8: The underside of the coil taped to the plastic lid 
was measured by manually determining the frequency peak using a Hewlett-Packard 3585A Spectrum Analyzer. The circuit was once again inductively coupled to the spectrum analyzer and the frequency was determined manually be observing the peak frequency.

A tank apparatus seen in Fig. 5.6 was set up for fixed volume measurements. A tank measuring $40 \mathrm{~cm}$ long and $20 \mathrm{~cm}$ wide was filled to $20 \mathrm{~cm}$ with $16 \mathrm{~L}$ of water. A $2000 \mathrm{~mL}$ plastic graduated cylinder was placed upside down in the tank on top of plastic blocks. A bag of sand was balanced as a weight on top of the cylinder to keep it in place. The water level was marked on the cylinder with black electrical tape. Tape markers were also placed $1 \mathrm{~L}$ above and $400 \mathrm{~mL}$ below the water level. A tube was taped to the inside of the cylinder for participants to breathe through. A disposable straw was attached at the other end and the tube was washed after each participant to prevent cross-contamination. As the participant breathed in and out through the straw, the water level in the cylinder rose and fell respectively. This experimental setup was chosen rather than using a pneumotach because of its simplicity and its ability to give the participants a visual indication of the volume of air they were breathing.

The Colpitts oscillator measurements were taken first for each participant. Each of the three procedures was done at least three times and no more than four times. For the first procedure the participant breathed out fully to residual volume, breathed in fully to total lung capacity, and breathed out again to residual volume. The participant held his or her breath for several seconds at the maximal points while each measurement was taken. The participant wore a nose plug for the next two procedures in order to ensure that all breathing was through the mouth. In the second procedure, the participant breathed out to residual volume, breathed in through the straw to the $1 \mathrm{~L}$ mark, and breathed out through the straw to the original water level 
mark. Once again, the participant held his or her breath for several seconds at the three points and maintained the high water mark by plugging the straw with his or her tongue to allow for slight relaxation of the lungs. For the third procedure, the participant breathed out through the straw to the $400 \mathrm{~mL}$ mark, breathed in through

the straw to the original water mark, and breathed out again through the straw to the $400 \mathrm{~mL}$ mark. The participant held their breath and plugged the straw again at the three points. These three procedures were repeated using the relaxation oscillator circuit.

\subsubsection{In Vivo Trial Results}

The average oscillating frequency of the Colpitts circuit was $2.31 \mathrm{MHz}$ and the relaxation circuit had an average frequency of $4.057 \mathrm{MHz}$. The frequency span on the analyzer was set to $5 \mathrm{kHz}$ for the measurements with the Colpitts circuit and $50 \mathrm{kHz}$ with the relaxation circuit. The measurement error of the Colpitts circuit was \pm 20 $\mathrm{Hz}$ and the relaxation circuit measurements had an error of $\pm 200 \mathrm{~Hz}$.

Fig. 5.9 shows the average frequency change of the Colpitts circuit as the participant breathes in and out for the three different procedures. Fig. 5.10 shows the equivalent for the relaxation circuit. Each subplot shows the average from each participant as well as the average over all participants. For a given participant, a baseline was established by subtracting the average first frequency measured from the next two averaged measurements so that the data are presented as the change in frequency from the first measurement. Error bars were estimated from measurement errors.

Table 5.4 gives the theoretical change in impedance and relative change in frequency we predict for the in vivo trials. We use the following values to predict the relative change in frequency: the coil radius $R=4.6 \mathrm{~cm}$, the number of turns $N=10$, the distance between coil and centre of lung $z_{0}=10 \mathrm{~cm}$, the change in conductivity 


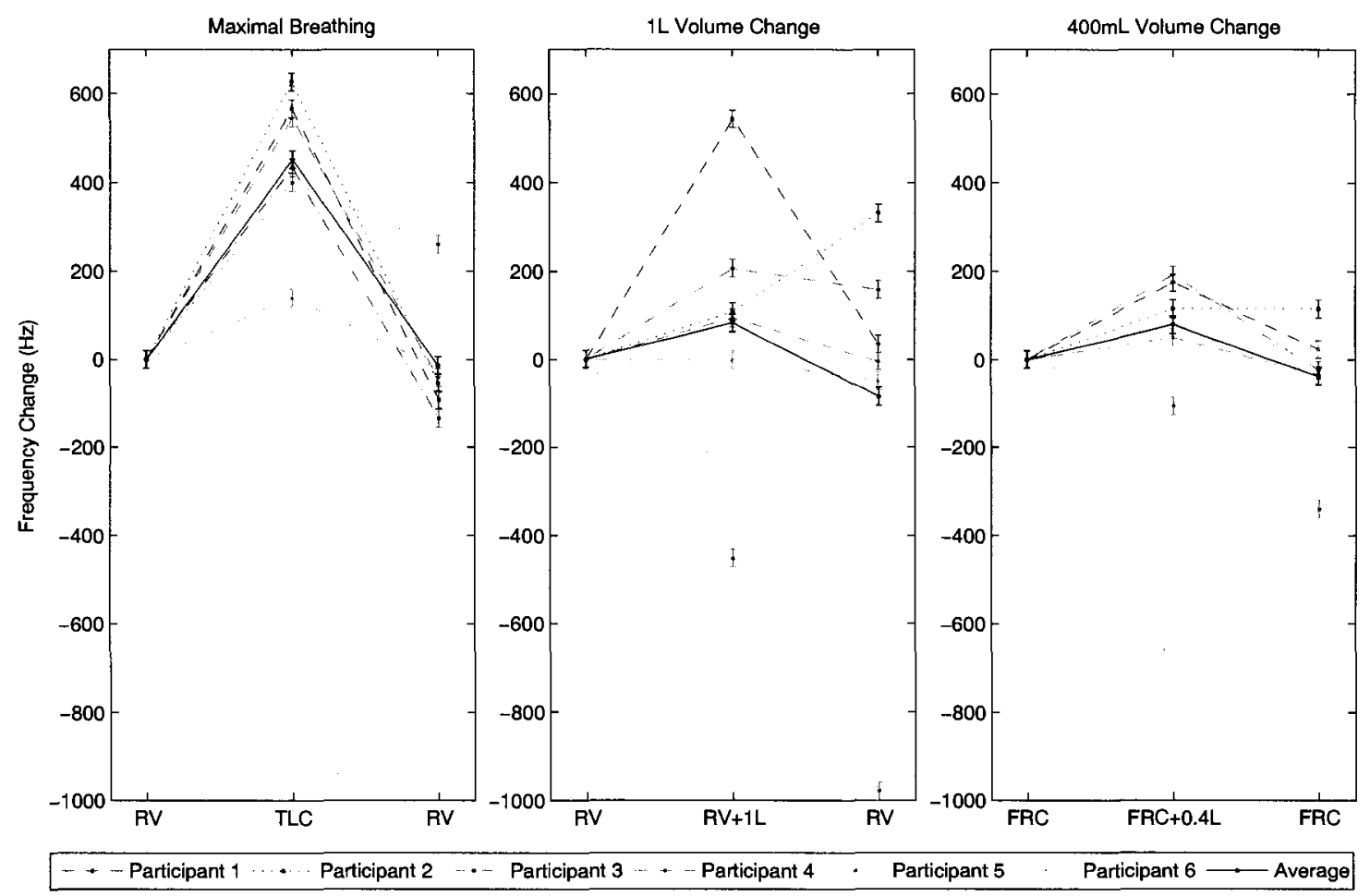

Figure 5.9: The results of the in vivo trials using the Colpitts circuit 


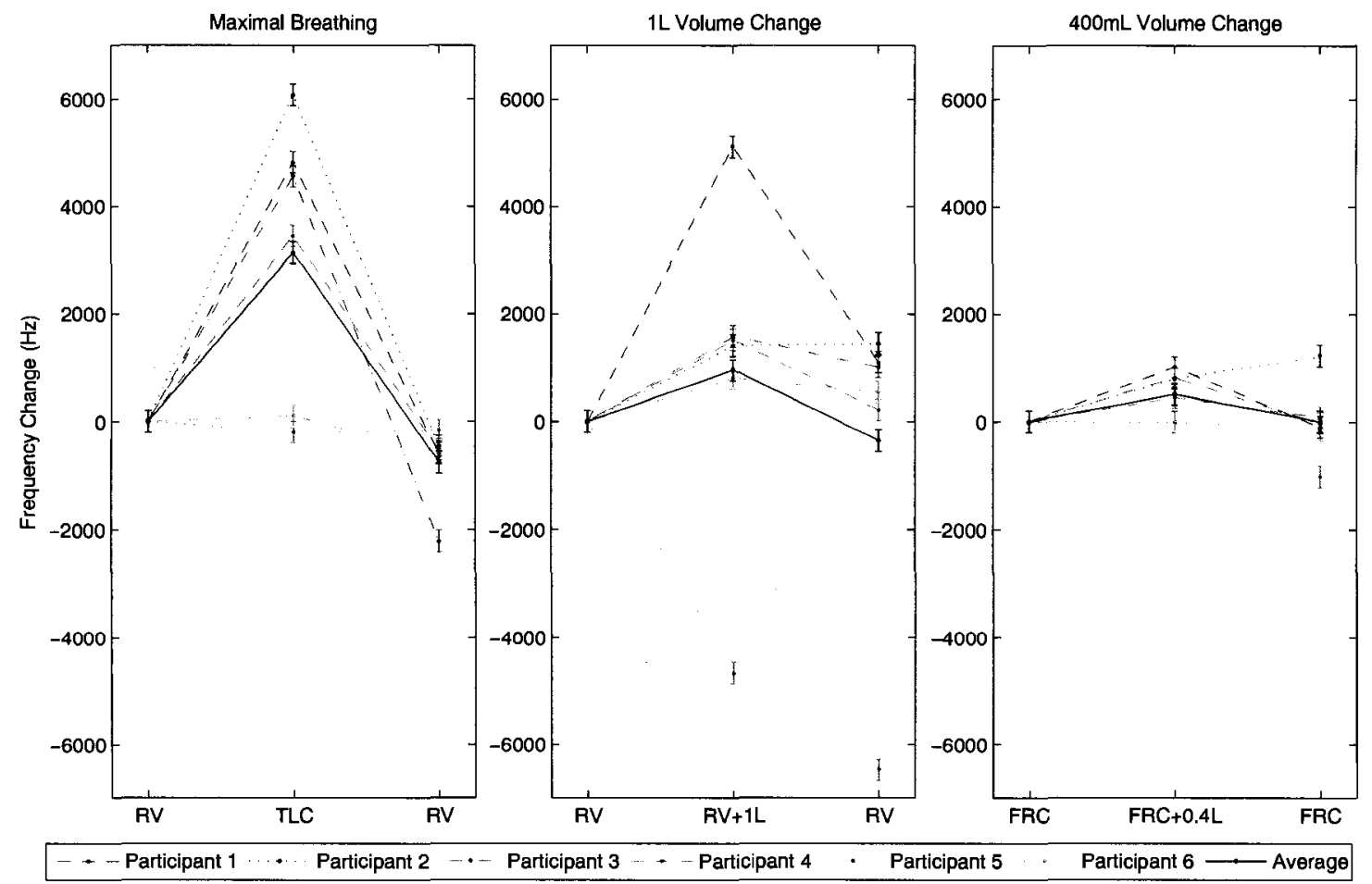

Figure 5.10: The results of the in vivo trials using the relaxation circuit 


\begin{tabular}{|l||c|c||c|c||c|c|}
\hline \multicolumn{1}{|c||}{} & \multicolumn{2}{c||}{ TLC } & \multicolumn{2}{c|}{ 1 Litre } & \multicolumn{2}{c|}{ 400 mL } \\
\cline { 2 - 7 } & $\Delta Z(m \Omega)$ & $\frac{\Delta f}{f} \cdot 10^{6}$ & $\Delta Z(m \Omega)$ & $\frac{\Delta f}{f} \cdot 10^{6}$ & $\Delta Z(m \Omega)$ & $\frac{\Delta f}{f} \cdot 10^{6}$ \\
\hline Colpitts & 28.0 & 6.99 & 1.9 & 0.47 & 0.5 & 0.13 \\
\hline Relaxation & 86.2 & 261 & 5.8 & 18 & 1.6 & 5 \\
\hline
\end{tabular}

Table 5.4: The theoretical change in impedance $\Delta Z$ and relative change in frequency $\frac{\Delta f}{f}$ that our model predicts for the in vivo trials. We use $R=4.6 \mathrm{~cm}, N=10$, $z_{0}=10 \mathrm{~cm}, \Delta \sigma=0.21 \mathrm{~S} / \mathrm{m}, f=2.31 \mathrm{MHz}$ and $R_{1}=10 \mathrm{k} \Omega$ for the Colpitts circuit, and $f=4.057 \mathrm{MHz}$ and $R_{1}=330 \mathrm{k} \Omega$ for the relaxation circuit.

\begin{tabular}{|l|c||c|c||c|c||c|c|}
\hline \multicolumn{2}{|c||}{} & \multicolumn{2}{c||}{ TLC } & \multicolumn{2}{c||}{ 1 Litre } & \multicolumn{2}{c|}{ 400 $\mathbf{~ m L}$} \\
\cline { 3 - 8 } \multicolumn{2}{|c|}{} & $\bar{x}$ & $\sigma$ & $\bar{x}$ & $\sigma$ & $\bar{x}$ & $\sigma$ \\
\hline \multirow{3}{*}{ Colpitts } & $\Delta f(\mathrm{~Hz})$ & 469 & 210 & 124 & 190 & 99 & 73 \\
\cline { 2 - 8 } & $\frac{\Delta f}{f} \cdot 10^{6}$ & 203 & 91 & 54 & 82 & 43 & 32 \\
\hline \multirow{2}{*}{ Relaxation } & $\Delta f(\mathrm{~Hz})$ & 3517 & 2820 & 1139 & 2428 & 510 & 405 \\
\cline { 2 - 8 } & $\frac{\Delta f}{f} \cdot 10^{6}$ & 869 & 698 & 280 & 600 & 126 & 100 \\
\hline
\end{tabular}

Average Frequency Changes 5.5: The averages and standard deviations of frequency changes from the lung manoeuvers measured in $\mathrm{MHz}$ with the two different circuits.

$\Delta \sigma=\sigma_{d}-\sigma_{i}=0.21 \mathrm{~S} / \mathrm{m}$, the Colpitts circuit average frequency $\bar{f}=2.31 \mathrm{MHz}$, the relaxation circuit average frequency $\bar{f}=4.057 \mathrm{MHz}$, the resistors $R_{1}=10 \mathrm{k} \Omega$ for the Colpitts circuit and $R_{1}=330 \mathrm{k} \Omega$ for the relaxation circuit.

\subsubsection{Statistical Analysis}

The average change in frequency and relative change in frequency parameters were calculated for the two circuits and the three different procedures and are shown in Table 5.5. Note that all data were used in establishing the average and standard deviation even though some participants had more than three trials for some procedures.

A paired T-test was performed with the data in order to establish whether or not the results could be explained by noise. Each line of data was converted to one change of frequency value. In other words, we averaged the first and last frequency 


\begin{tabular}{|l|c|c|c|}
\hline & TLC & 1 Litre & $\mathbf{4 0 0} \mathbf{~ m L}$ \\
\hline Colpitts & $5.34 \cdot 10^{-9}$ & $8.7 \cdot 10^{-3}$ & $2.26 \cdot 10^{-5}$ \\
\hline Relaxation & $5.99 \cdot 10^{-5}$ & $6.29 \cdot 10^{-2}$ & $5.41 \cdot 10^{-5}$ \\
\hline
\end{tabular}

Table 5.6: The probability that the measured frequency changes could be explained by noise. The null hypothesis is rejected in trials except the $1 \mathrm{~L}$ trial with the relaxation coil

measurement, both of these points are when the participant has exhaled. We then take the change between the second measurement, which is when the participant has inhaled, and the averaged value exhale value is:

$$
f_{2}-\frac{f_{1}+f_{3}}{2} .
$$

The measurements from the trials provided at least 17 degrees of freedom for each trial. Table 5.6 lists the probability that the frequency changes measured could be explained by noise. The T-test revealed that with a significance level of $5 \%$, the null hypothesis was rejected in all but one trial. The $1 \mathrm{~L}$ trials using the relaxation circuit had a $6.29 \%$ probability, which was due to the measurements from one participant. Referring to Fig. 5.10, we see that participant \#5 showed very different behaviour than the other participants in the $1 \mathrm{~L}$ breathing trials with the relaxation circuit. If these outliers are removed, and the T-test is performed with only 14 degrees of freedom, we get a probability of $0.14 \%$. We are therefore confident that the frequency changes we have measured in the in vivo trials are significant.

\subsubsection{Discussion of In Vivo Trial Results}

Even after considering that the relaxation circuit operates at almost twice the frequency of the oscillator circuit, the relaxation still gives a much larger relative change in frequency. However, there was significant measurement error in the relaxation 
circuit, particularly relative to the $400 \mathrm{~mL}$ breathing manoeuvers. From a practical perspective the Colpitts circuit had a much clearer, more defined resonance frequency peak, which gave it a higher resolution.

There are several inaccuracies and sources of error from these measurements in addition to those listed in the results. Firstly, the coil was positioned roughly under the right lung of each participant, but no fiducial markers were used, so the coil would have been centred better under some participants than others. A large source of error is movement of the body during breathing. Participants were asked to lay as still as possible, but there was still some movement, particularly from the participants' arms as these were needed to hold the straw. The coil was housed in a lid to protect it and keep it from bending, but it is possible that the coil could still have experienced some pressure differences as participants breathed.

The height difference on the cylinder for $1 \mathrm{~L}$ is $21.0 \mathrm{~cm}$ and it is $8.2 \mathrm{~cm}$ for 400 $\mathrm{mL}$. These values are not proportional because the cylinder's radius changes slightly from top to bottom. The breathing tube had a $1 \mathrm{~cm}$ diameter, so by modeling the tube as a cylinder with a $0.5 \mathrm{~cm}$ radius,

$$
\begin{aligned}
v & =\pi r^{2} h \\
v_{1 L} & =\pi(.5)^{2}(21)=16.5 \\
v_{400 m L} & =\pi(.5)^{2}(8.2)=6.4,
\end{aligned}
$$

we see that the $1 \mathrm{~L}$ breathing manoeuvers were approximately $16.5 \mathrm{~mL}$ shy of $1 \mathrm{~L}$ and the $400 \mathrm{~mL}$ breathing manoeuvers were approximately $6.4 \mathrm{~mL}$ less than $400 \mathrm{~mL}$. The participants tried to breathe as close as possible to the markers and the error was within $\pm 20 \mathrm{~mL}$.

The volume measurements required participants to effectively lift or push down 
a column of water which meant that the air was not at atmospheric pressure. The water level in the tank changes as water is sucked up the cylinder or air is pushed down. For the $1 \mathrm{~L}$ breathing, the water level decreases by about $1.3 \mathrm{~cm}$, making the height difference from the top of the water column to the water level in the tank 22.3 $\mathrm{cm}$. For the $400 \mathrm{~mL}$ breathing, the water level increases by about $0.5 \mathrm{~cm}$, making the height difference from the water level in the tank to the bottom of the column of air $8.7 \mathrm{~cm}$.

Hydrostatic pressure is calculated as follows:

$$
P_{\text {top }}=P_{\text {bottom }}-\rho g h
$$

where $\rho$ is the density of water, $g$ is the acceleration of gravity, and $h$ is the height difference. We assume that the room is at atmospheric pressure and the water is at about $22{ }^{\circ} \mathrm{C}$, giving it a density $998 \mathrm{~kg} / \mathrm{m}^{3}$. We use Eqn. 5.6 to calculate the pressure at the top of the water column for the $1 \mathrm{~L}$ breathing manoeuver and after taking into account the accuracy of the height of the column, we can say that the participant has to generate an underpressure of $2.18 \pm 0.05 \mathrm{kPa}$ with their pleural muscles. Similarly, we calculate the pressure at the bottom of the $400 \mathrm{~mL}$ air column and conclude that the participant must generate an overpressure of $0.85 \pm 0.05 \mathrm{kPa}$ to effect this manoeuver. This is possible due to the compliance of the lungs and the difference between the lung volume and the volume change seen in the cylinder can be calculated:

$$
\Delta V_{\text {lungs }}=\Delta V_{\text {cylinder }}+\frac{\Delta P}{C_{\text {lungs }}}
$$

where $\Delta \mathrm{P}$ is the pressure generated by the lungs and $C_{\text {lungs }}$ is the lung compliance. However, since the participant was plugging the straw at the times of measurement, the chest muscles were relaxed and there were no muscular movements during the 
measurements. Thus, $\Delta P=0$, and $\Delta V_{\text {lungs }}=\Delta V_{\text {cylinder }}$.

We assume that the air in both the lungs and the cylinder is humid but that the air in the cylinder is at about $22{ }^{\circ} \mathrm{C}(295 \mathrm{~K})$, whereas the air in the lungs is about 37 ${ }^{\circ} \mathrm{C}(310 \mathrm{~K})$. As the participant breathes in air from the cylinder, the air is heated by the body in an isobaric process,

$$
V_{2}=V_{1} \frac{T_{2}}{T_{1}}
$$

For the given temperature change, the air in the lungs expands by about $5 \%$ to 1.051 L. The participant breathes out warm air which mixes with the air that was in the cylinder and the tube from the mouth of the participant to the cylinder and a final temperature, volume, and pressure are reached. We say that the participant breathes out $1 \mathrm{~L}$ of air at atmospheric pressure and therefore the air that was in the cylinder and tube is also at atmospheric pressure. After the two sources mix it turns out that the volume and pressure of the air is the same as the initial volume and pressure.

We can check this by first noting that the change in internal energy $\Delta U$ of the system is zero and there is no work done on the system. The participant breathes out $V_{L}=1 \mathrm{~L}$ of air from the lungs at $T_{L}=310 \mathrm{~K}$. There is $600 \mathrm{~mL}$ of air in the cylinder and an additional $50 \mathrm{~mL}$ in the tube and this is at $T_{C}=295 \mathrm{~K}$. We can thus 
calculate the final temperature $T_{F}$ of the mixed air,

$$
\begin{aligned}
0 & =\Delta U_{L}+\Delta U_{C} \\
0 & =\alpha R n_{L} \Delta T_{L}+\alpha R n_{C} \Delta T_{C} \\
0 & =\frac{P_{L} V_{L}}{T_{L}} \Delta T_{L}+\frac{P_{C} V_{C}}{T_{C}} \Delta T_{C} \\
0 & =\frac{V_{L}}{T_{L}}\left(T-T_{L}\right)+\frac{P_{C} V_{C}}{T_{C}}\left(T-T_{C}\right) \\
T_{F} & =\frac{T_{C} T_{L}\left(V_{L}+V_{C}\right)}{T_{C} V_{L}+T_{L} V_{C}} \\
T_{F} & =\frac{(295)(310)((1)+(.65))}{(295)(1)+(310)(.65)} \\
T_{F} & =304 K
\end{aligned}
$$

where $R$ is the ideal gas constant, $n$ is the number of moles, $\alpha$ is the degrees of freedom of the gas. The total number of moles of air in the system does not change and we assume that the final pressure is atmospheric,

$$
\begin{aligned}
n_{F} & =n_{L}+n_{C} \\
\frac{P_{F} V_{F}}{R T_{F}} & =\frac{P_{L} V_{L}}{R T_{L}}+\frac{P_{C} V_{C}}{R T_{C}} \\
V_{F} & =T_{F}\left(\frac{V_{L}}{T_{L}}+\frac{V_{C}}{T_{C}}\right) \\
V_{F} & =(304)\left(\frac{(1)}{(310)}+\frac{(.65)}{(295)}\right) \\
V_{F} & =1.65 L
\end{aligned}
$$

The final volume is the sum of $V_{L}$ and $V_{C}$.

To summarize, this means that during the trials, the participant breathes out to residual volume, breathes in $1 \mathrm{~L}$, which expands to $1.051 \mathrm{~L}$, then breathes out $1 \mathrm{~L}$ leaving $51 \mathrm{~mL}$ in addition to the residual volume. This is a relatively insignificant 
amount and we can ignore it. The same arguments would be applied to the case of the $400 \mathrm{~mL}$ breathing trials. We have ignored the effect of respiratory exchange in this analysis as the lungs absorb oxygen and expel carbon dioxide.

Participants were not restricted in their breathing mechanism, so we have no indication whether the participants were performing thoracic or abdominal breathing. While the breathing mode should affect the results, it makes more sense to leave this as an unknown since the sensor is meant to be noncontact.

\section{$5.3 \quad$ Summary}

This chapter presented the phantom trials and in vivo experiments that we conducted to test the theoretical model and the sensitivity of the coil sensor. The experimental setup and results were presented for all trials and a discussion of the experiments followed. While the results did not match perfectly with the theoretical derivation, we were able to see statistically significant changes in frequency with the sensor. 


\section{Chapter 6}

\section{Discussion}

\subsection{Summary of Contributions}

Current techniques for respiratory monitoring and were researched, with particular emphasis on those with applications to sleep and apnea monitoring, and we focused on single coil electromagnetic sensors. We developed a theoretical model to predict the changes that a single coil sensor driven by an oscillating current would experience from conductivity variations of the thorax caused by respiration. Respiratory activity was modeled as a homogeneous sphere located on axis above the coil. The change in conductivity of the sphere was taken as the difference in conductivity between an inflated and deflated lung. The radius of the sphere was determined by the lung volume and the effect was seen as a change in coil impedance.

We investigated several oscillator circuits to implement our sensor and eventually pursued two different circuit designs. The circuits were analyzed and we calculated the conductivity induced changes in frequency of the oscillators by treating the theoretical change in coil impedance as an effective resistor in series with the coil. This theoretical model was then used to optimize the sensitivity of the sensor through 
circuit component choice.

We tested the sensitivity of both circuits with phantom trials and in vivo trials. The phantom trials used a cylindrical rather than spherical conductive volume to analyze how well our theoretical model correlated to measurements as well as to evaluate the sensitivity of the sensor. The in vivo trials were conducted to determine the feasibility of using the electromagnetic sensor to monitor breathing and to further test the sensitivity of the sensor.

\subsection{Comparison of Model and Experimental Re- sults}

Our phantom trials showed some correlation between the theoretical model and the sensor measurements. Plots of the relative change in frequency demonstrated that theoretical and measured results had similar curves, but the magnitude of the curves did not always match well. A particularly odd result was that the relative change in frequency of the sensor did not appear to be directly proportional to conductivity as expected. This suggests that there are other factors influencing the sensor's performance.

The in vivo trials demonstrated that both electronic designs of the sensor were able to detect changes in lung volume. For several participants, the magnitude of the change in frequency corresponded roughly to the volume of air displaced which suggests that a similar sensor could be calibrated to estimate changes in lung volume. A paired T-test was performed on the measurements and with the exception of some outliers, the results indicated that the changes in frequency measured by the sensor were unlikely to be caused by noise. 


\subsection{Conclusions}

The single coil electromagnetic sensor has been shown to detect conductivity changes due to respiratory activity and its sensitivity has been analyzed. Since the phantom trials did not show complete agreement with the theoretical model, further research is needed to fully validate the theoretical model. Efforts were made to maximize the sensitivity of the coil and to limit outside influences such as temperature drifts due to air currents and conductive objects in the vicinity of the testing area, but the phantom trials suggested that there may still have been factors influencing measurements.

\subsection{Future Research}

Further to the research presented in this thesis and given the results obtained from the phantom and in vivo trials in particular, the following recommendations for future research are made:

- Using the theoretical model as a guide, different circuit components could be selected and the circuits tested with conductive phantoms

- The changes in conductivity of a lung could be modeled over the volume of a spherical shell rather than a solid sphere since lungs always retain a residual volume of air

- The changes in conductivity of a lung could be modeled as an infinite conductive plane with a variable thickness

- The inherent impedance of the coil could be integrated into the frequency calculations to provide a more accurate model of circuit behaviour 
- The permittivity changes could be included in the theoretical model, giving a complex impedance $\Delta \mathrm{Z}$ which would also change the circuit analysis and model

- The electronic circuit could be extended in order to output a continuous signal with an amplitude proportional to volume or conductivity changes which would allow for analysis of breathing without breath holds.

- Since the theoretical change in impedance of the coil is dependent on the oscillator frequency, a system for maintaining a constant frequency could be integrated to give more linear results as presented by Guardo et al [12]

- Multiple coils arranged in an array could provide a more complete picture of conductivity changes which would be less dependent on the positioning of the coil 


\section{Appendix A}

\section{A.1 Matlab Code}

function $v=$ sphere $\left(R, r \_0, z_{-} 0\right)$

$\%$ Calculates the change in impedance of a coil from a conductive sphere $\% \mathrm{R}$ is the coil radius in metres

$\% \mathrm{r} \_0$ is the sphere radius in metres

$\% \mathrm{z}_{-} 0$ is the distance between coil and centre of sphere in metres

warning('off,'MATLAB:quad:MinStepSize') \% turn off warnings warning('off,'MATLAB:divideByZero') \% turn off warnings

$\%$ fundamental constants used in fn9 and fin 6

$\% \mathrm{mu} 0=4 * \mathrm{pi}^{*} 10^{\wedge}(-7) ; \%$ permeability of free space (N/A^2)

$\%$ epsilon_0 $=8.85^{*} 10^{\wedge}(-12) ; \%$ permittivity of free space $\left(\mathrm{C}^{\wedge} 2 /\left(\mathrm{N}^{*} \mathrm{~m}^{\wedge} 2\right)\right)$

$\%$ measurement setup and tissue parameters

$\%$ sigma $=.21 ; \%$ change in lung tissue conductivity $(\mathrm{S} / \mathrm{m})$ used in fn6

$\%$ epsilon=epsilon_0; \% permittivity of tissue $\left(\mathrm{C}^{\wedge} 2 /\left(\mathrm{N}^{*} \mathrm{~m}^{\wedge} 2\right)\right)$ used in fn6

$\mathrm{N}=10 ; \%$ of turns in coil,also used in fn8

$\mathrm{f}=2.31 * 10^{\wedge} 6$; \% frequency of Colpitts circuit $(\mathrm{Hz})$ also used in $\mathrm{fn} 6$ $\% \mathrm{f}=4.057 * 10^{\wedge} 6 ; \%$ frequency of Relaxation circuit $(\mathrm{Hz})$ also used in fn6

omega $=2 *$ pi*f; $\%$ angular frequency (radians/s) also used in fn6

$\%$ Multiply our results of integration by the missing constants from fn6, $\% \mathrm{fn} 7$, and fn9.

$\mathrm{v}=-\mathrm{omega}^{\wedge} 2 * \mathrm{~N} * \mathrm{fn} 1\left(\mathrm{R}, \mathrm{r} \_0, \mathrm{z} \_0\right) ; \%$ impedance change 
function $v=f n 1\left(R, r \_0, z \_0\right) \%$ Magnetic flux part1

$\%$ This is the angular integration across the area of interest for the $\%$ magnetic flux calculation. The magnetic field does not depend on angle, $\%$ therefore the integral is simply 2 pi.

$\mathrm{v}=2 * \mathrm{pi}^{*} \mathrm{fn} 2\left(\mathrm{R}, \mathrm{r} \_0, \mathrm{z} \_0\right) ; \%$ multiply function by $2 \mathrm{pi}$

function $v=f n 2\left(R, r \_0, z \_0\right) \%$ Magnetic flux part2

$\%$ This is the radial integration across the area of interest for the

$\%$ magnetic flux calculation. The area is circular, therefore the radial

$\%$ integration must be rdr, hence the multiplication by s'.

$\lim 1=0$

$\lim 2=\mathrm{R}$;

\%integrate over s prime from 0 to $\mathrm{R}$

$\mathrm{v}=$ quad(@(s_prime) $\left(\mathrm{s} \_\right.$prime. ${ }^{*} \mathrm{fn} 3\left(\mathrm{~s} \_\right.$prime,R,r_0,z_0 $\left.\left.)\right), \lim 1, \lim 2\right)$;

function $v=f n 3$ (s prime,R,r_0,z_0) \% Magnetic field part1

$\%$ This is the axial integration over the height of the conductive object

$\%$ for the magnetic field calculation. For a sphere $\lim 1=\mathrm{z} 0-\mathrm{r} 0$ and

$\% \lim 2=z_{-} 0+r_{-} 0$ where $z_{-} 0$ is the height of the centre of the sphere and $\% \mathrm{r}[0$ is the radius of the sphere.

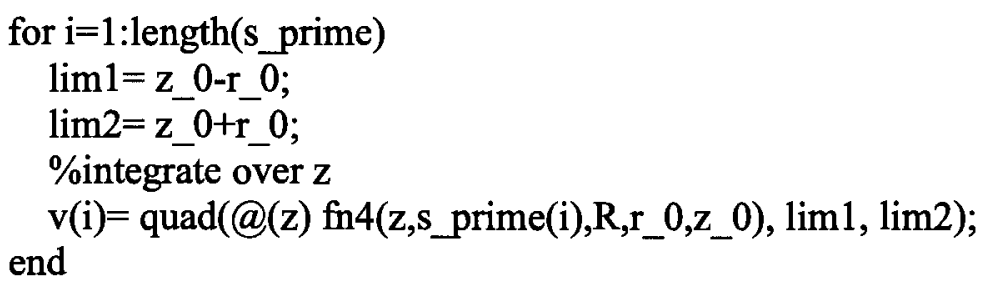

function $v=f n 4(z, s$ prime,R,r_0,z_0) $\%$ Magnetic field part 2

$\%$ This is the radial integration across the conductive disk for the magnetic $\%$ field calculation. The disk radius $\lim 2$ is a function of $\mathrm{z}$ for a

$\%$ sphere, but is constant for a cylinder. $\lim 2=\left(r \_0^{\wedge} 2-\left(z \_0-z(i)\right)^{\wedge} 2\right)^{\wedge}(1 / 2)$

$\%$ for a sphere where $z_{-} 0$ is the height of the centre of the sphere.

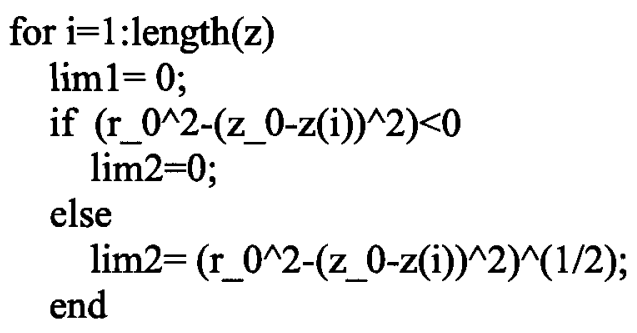

\%integrate over rho from 0 to $\lim 2$

$\mathrm{v}(\mathrm{i})=$ quad(@(rho) fn5(rho,z(i),R,s_prime), lim1, lim2); end 
function $v=f n 5(r h o, z, R, s$ prime $) \%$ Current density and magnetic field terms $\%$ This is the function that gets integrated by drhodz. The first term is $\%$ the current density at a point (rho,z) created by a loop of radius $\mathrm{R}$, the $\%$ second term is the magnetic field that a loop (of radius rho at height $\mathrm{z}$ ) $\%$ of this current produces at a point $\left(s^{\prime}, 0\right)$.

\%multiply current density by magnetic field from a loop, set \#loops $\mathrm{N}=1$ $\mathrm{v}=\mathrm{fn} 6(\mathrm{rho}, \mathrm{z}, \mathrm{R}) .{ }^{*} \mathrm{fn} 9\left(\mathrm{~s} \_\right.$prime,z,rho,1);

function $v=$ fn6(rho,z,R) \% Current density

$\%$ The current density is the admittivity times the electric field so

$\% \mathrm{~J}=\mathrm{C} *$ fn6, where $\mathrm{C}=$ admittivity.

sigma $=.21 ; \%$ conductivity of tissue $\left(1 /\left(\mathrm{ohm}^{*} \mathrm{~m}\right)\right)$

epsilon_ $0=8.85^{*} 10^{\wedge}(-12) ; \%$ permittivity of free space $\left(\mathrm{C}^{\wedge} 2 /\left(\mathrm{N}^{*} \mathrm{~m}^{\wedge} 2\right)\right)$

epsilon $\bar{R}=0 ; \%$ change in relative permittivity of tissue $\left(C^{\wedge} 2 /\left(N^{*} m^{\wedge} 2\right)\right)$

$\mathrm{f}=2.31 * 10^{\wedge} 6 ; \%$ average frequency of Colpitts circuit $(\mathrm{Hz})$

$\% \mathrm{f}=4.057 * 10^{\wedge} 6 ; \%$ average frequency of Relaxation circuit $(\mathrm{Hz})$

omega $=2 * \mathrm{pi}^{*} \mathrm{f} ; \%$ angular frequency (radians $/ \mathrm{s}$ )

c_2=sigma $+\mathrm{j} *$ omega*epsilon_0*epsilonR; $\%$ tissue admittivity, $\mathrm{j}=(-1)^{\wedge}(1 / 2)$

$\mathrm{v}=\mathrm{c} \_2 * \mathrm{fn} 7(\mathrm{rho}, \mathrm{z}, \mathrm{R})$

function $v=f n 7($ rho,z,R) $\%$ Electric field partl

$\%$ This is the angular integration across the area of interest for the

$\%$ electric field calculation. The magnetic field does not depend on angle,

$\%$ therefore the integral is simply $2 \mathrm{pi}$. However, the loop integral of

$\%$ electric field is $2 \mathrm{pi}^{*}$ rho, so the 2 pis cancel and we're left with $1 /$ rho.

$\%$ The negative is due to the negative in Faraday's law

$\%$ So $E=I^{\prime *}$ fn 7

$\mathrm{v}=-\mathrm{fn} 8(\mathrm{rho}, \mathrm{z}, \mathrm{R}) . / \mathrm{rho} ; \%$ multiply function by $-2 \mathrm{pi}$, divide by $2 \mathrm{pi}{ }^{*}$ rho

function $v=f(n 8($ rho, $z, R) \%$ Electric field part 2

$\%$ This is the radial integration across the area of interest for the

$\%$ electric field calculation. The area is circular, therefore the radial

$\%$ integration must be rdr, hence the multiplication by s.

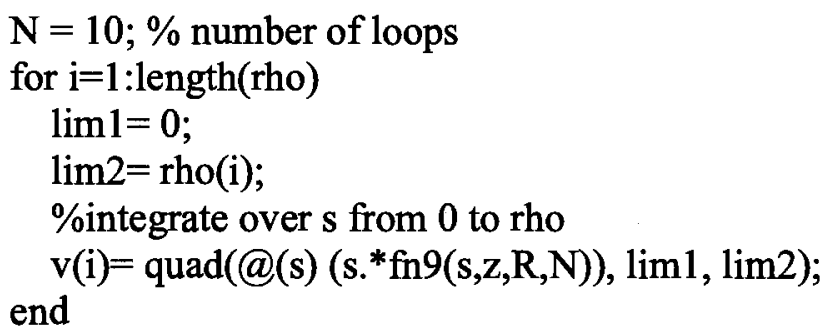


function $v=f(s, \mathbf{s}, \mathbf{Z}, \mathrm{R}, \mathrm{N}) \%$ magnetic field from loop current source $\%$ This is the $z$-hat component of the magnetic field from a loop of current $\%$ at point $(\mathrm{s}, \mathrm{z})$, where $\mathrm{S}=$ radius of point from $\mathrm{z}$-axis, $\mathrm{Z}=$ height of point, $\% R$-radius of loop, $B \_z(s, z, t)=C^{*} I^{*} f n 9$, where $C=m u \_0 * N /(2 p i)$

$\%$ This function was obtained by symbolically integrating $\% R^{*}(\mathrm{R}-\mathrm{s} \cos (\mathrm{phi})) \mathrm{d}(\mathrm{phi}) /\left(\mathrm{s}^{\wedge} 2+\mathrm{R}^{\wedge} 2+\mathrm{z}^{\wedge} 2-2 \mathrm{sR} \cos (\mathrm{phi})\right)^{\wedge}(3 / 2)$ over angle phi $\%$ from 0 to 2 pi. The result is a function of elliptical integrals.

$\%$ Assume permeability is constant at mu_ $0=4 * \mathrm{pi}^{*} 10^{\wedge}(-7)\left(\mathrm{N} / \mathrm{A}^{\wedge} 2\right)$, the $\%$ permeability of free space.

$a=\left(s . \wedge 2+R .{ }^{\wedge} 2+z^{\wedge} 2-2 * s * R\right) ; \%$ simplify function into larger variables $\mathrm{b}=\left(\mathrm{s} . \wedge 2+\mathrm{R},{ }^{\wedge} 2+\mathrm{z}^{\wedge} 2+2^{*} \mathrm{~s}^{*} \mathrm{R}\right) ; \%$ simplify function into larger variables

$\%$ evaluate the complete elliptic integrals $\left.[\mathrm{c}, \mathrm{d}]=\operatorname{ellipke}\left(2^{*}\left(\mathrm{~s}^{*} \mathrm{R} . / \mathrm{b}\right)\right)^{\wedge}(1 / 2)\right)$; $\mathrm{v}=2 \mathrm{e}-7^{*} \mathrm{~N}^{*}\left((\mathrm{a} . / \mathrm{b}) .^{\wedge}(1 / 2)\right){ }^{*}\left(\mathrm{a} .{ }^{*} \mathrm{c}-\left(\mathrm{s} .{ }^{\wedge} 2+\mathrm{z}^{\wedge} 2-\mathrm{R} .{ }^{\wedge} 2\right) .{ }^{*} \mathrm{~d}\right) . / \mathrm{a} \cdot{ }^{\wedge}(3 / 2)$ 


\title{
Appendix B
}

\author{
B.1 Letter of Information and Consent
}


Letter of Information and Consent

Lung Monitoring Using a Contactless Electromagnetic Sensor

Code:

\section{Researchers}

Rosalyn Seeton, MASc. Biomedical Engineering Candidate

Systems and Computer Engineering, Carleton University

seeton@sce.carleton.ca

520-2600 ext.1074, Minto 6070

Dr. Andy Adler, Supervisor

Systems and Computer Engineering, Carleton University

adler@sce.carleton.ca

520-2600 ext.8785, Minto 7040

Researchers may be contacted by phone or email should a participant have any questions or wish to withdraw from the study.

\section{Purpose}

This is a research study to examine the feasibility of non-invasive lung monitoring using a novel technique: a contactless electromagnetic sensor. This study has applications such as sleep monitoring and has the potential to significantly make improvements to the current state of the art.

\section{Procedures}

The measurements will be made in a lab with restricted access to ensure maximum privacy and the measurements should take less than half an hour. An electromagnetic coil sensor will be positioned near the chest. The participant will be asked to perform breathing manoeuvers with breath holds (breathe normally, breathe in as much as possible, breathe out as much as possible, breathe a certain volume). The participants will breathe into a tube connected to a tank of water to visually identify the volume of air. The participant's age and gender will be recorded as part of the data collection. The participant will be supervised at all times while measurements are acquired.

\section{$\underline{\text { Risks and Benefits }}$}

There is the possibility of minor physical discomfort from the measurement setup while conducting study. Subjects who have known heart or lung conditions are asked not to participate in the study because participants will be asked to make breathing manoeuvers which may be difficult.

The only potential benefit to the participant is the possibility of advancement in measurement techniques in the long term.

\section{Security and Anonymity of Data}

Data from all participants will be kept secure and all publications based on these data will preserve their anonymity. Participants will be assigned a code with their consent form. The data will be recorded using this code. Consent forms will be stored in locked cabinets to ensure anonymity. Soft copies of data will be kept locked and password protected. 
Digital pictures may be taken of one or more participants in order to demonstrate the measurement setup and tasks. Pictures will only be taken if participants consent to it. Faces will be concealed so that participants cannot be identified (unless otherwise permitted by the participant) and pictures will be recorded with the code from the consent form. Pictures will be kept locked and password protected for one year as they may be needed for publications. All soft and hard copies of the original pictures will be destroyed after one year unless otherwise permitted by the participant.

\section{Withdrawal}

Participants may withdraw at any time if they so choose. Any data or information from a participant that has withdrawn will be destroyed unless otherwise permitted by the participant.

\section{Research Findings}

Participants will have access to publications and presentations that result from the research. A participant may also view his/her data and information, but will not have access to those of others.

\section{Ethics Committee}

This project was reviewed and received ethics clearance by the Carleton University Research Ethics Committee. Participants with concerns or questions about their involvement in the study may contact the ethics committee chair:

Leslie MacDonald-Hicks

Carleton University Research Ethics Committee

Room 510B Tory, Carleton University

Phone: 613-520-2517

E-mail: ethics@carleton.ca

\section{Researchers}

Rosalyn Seeton

Dr. Andy Adler

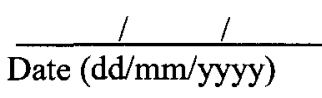

$\frac{1}{\text { Date }(\mathrm{dd} / \mathrm{mm} / \mathrm{yyyy})}$

\section{Consent}

I, have read the above letter and understand that I am participating in a research project and I voluntarily agree to participate.

I voluntarily agree to have my picture taken and included in publications and presentations.

— I do not agree to have my picture taken and included in publications and presentations. 


\section{References}

[1] T. R. Albrecht, P. Grütter, D. Horne, and D. Rugar. Frequency modulation detection using high-q cantilevers for enhanced force microscope sensitivity. Journal of Applied Physics, 69(2):668-673, 1991.

[2] Daniele Andreuccetti, Roberto Fossi, and Caterina Petrucci. Dielectric properties of body tissues. http://niremf.ifac.cnr.it/tissprop/, accessed December 11th, 2008.

[3] D. Barschdorff and Wei Zhang. Respiratory rhythm detection with photoplethysmographic methods. Engineering in Medicine and Biology Society, 1994. Engineering Advances: New Opportunities for Biomedical Engineers. Proceedings of the 16th Annual International Conference of the IEEE, pages 912-913 vol.2, 1994.

[4] K.P. Cohen, W.M. Ladd, D.M. Beams, W.S. Sheers, R.G. Radwin, W.J. Tompkins, and J.G. Webster. Comparison of impedance and inductance ventilation sensors on adults during breathing, motion, and simulated airway obstruction. Biomedical Engineering, IEEE Transactions on, 44(7):555-566, July 1997.

[5] Fairchild Semiconductor Corporation. Cmos oscillators. Application Note 118, Fairchild Semiconductor, Oct 1974. 
[6] J. Fei and I. Pavlidis. Analysis of breathing air flow patterns in thermal imaging. Engineering in Medicine and Biology Society, 2006. EMBS '06. 28th Annual International Conference of the IEEE, pages 946-952, 30 2006-Sept. 32006.

[7] M. Folke, L. Cernerud, M. Ekström, and B. Hök. Critical review of non-invasive respiratory monitoring in medical care. Med Biol Eng Comput, 41(4):377-383, July 2003.

[8] Camelia Gabriel and Sami Gabriel. Compilation of the dielectric properties of body tissues at rf and microwave frequencies. Report N.AL/OE-TR- 1996-0037, Occupational and environmental health directorate, Radiofrequency Radiation Division, Brooks Air Force Base, Texas (USA), June 1996.

[9] Camelia Gabriel, Sami Gabriel, and E Corthout. The dielectric properties of biological tissues: I. literature survey. Physics in Medicine and Biology, 41(11):2231$2249,1996$.

[10] Camelia Gabriel, R W Lau, and Camelia Gabriel. The dielectric properties of biological tissues: Ii. measurements in the frequency range $10 \mathrm{hz}$ to $20 \mathrm{ghz}$. Physics in Medicine and Biology, 41(11):2251-2269, 1996.

[11] Camelia Gabriel, R W Lau, and Camelia Gabriel. The dielectric properties of biological tissues: Iii. parametric models for the dielectric spectrum of tissues. Physics in Medicine and Biology, 41(11):2271-2293, 1996.

[12] R. Guardo, G. Charron, Y. Goussard, and P. Savard. Contactless measurement of thoracic conductivity changes by magnetic induction. Engineering in Medicine and Biology Society, 1997. Proceedings of the 19th Annual International Conference of the IEEE, 6:2450-2453 vol.6, Oct-2 Nov 1997. 
[13] R. Guardo, S. Trudelle, A. Adler, C. Boulay, and P. Savard. Contactless recording of cardiac related thoracic conductivity changes. Engineering in Medicine and Biology Society, 1995., IEEE 17th Annual Conference, 2:1581-1582 vol.2, Sep 1995.

[14] L.W. Hart, H.W. Ko, Jr. Meyer, J.H., D.P. Vasholz, and R.I. Joseph. A noninvasive electromagnetic conductivity sensor for biomedical applications. Biomedical Engineering, IEEE Transactions on, 35(12):1011-1022, Dec 1988.

[15] Megan Howell Jones, Rafik Goubran, and Frank Knoefel. Reliable respiratory rate estimation from a bed pressure array. Engineering in Medicine and Biology Society, 2006. EMBS '06. 28th Annual International Conference of the IEEE, pages 6410-6413, 30 2006-Sept. 32006.

[16] C H Igney, S Watson, R J Williams, H Griffiths, and O Dossel. Design and performance of a planar-array mit system with normal sensor alignment. Physiological Measurement, 26(2):S263-S278, 2005.

[17] Victor Koren. Rf oscillator uses current-feedback op amp. EDN, pages 83-84, Oct 2002 .

[18] A Korjenevsky, V Cherepenin, and S Sapetsky. Magnetic induction tomography: experimental realization. Physiological Measurement, 21(1):89-94, 2000.

[19] Russell L. Meade and Robert Diffenderfer. Foundations of Electronics: Circuits and Devices (Electron Flow Version). Thomson Delmar Learning, Clifton Park, NY, 5th edition, 2007. 
[20] Se Dong Min, Dae Joong Yoon, Sung Won Yoon, Yong Hyeon Yun, and Myoungho Lee. A study on a non-contacting respiration signal monitoring system using doppler ultrasound. Med Biol Eng Comput, 45(11):1113-1119, Nov 2007.

[21] Hiroaki Nakai, Ken Ishihara, Yoshio Miyake, and Mutsumi Watanabe. Nonrestrictive visual respiration monitoring. Pattern Recognition, International Conference on, 4:647-651, 2000.

[22] J Netz, E Forner, and S Haagemann. Contactless impedance measurement by magnetic induction - a possible method for investigation of brain impedance. Physiological Measurement, 14(4):463-471, 1993.

[23] Naresh M. Punjabi. The Epidemiology of Adult Obstructive Sleep Apnea. Proc Am Thorac Soc, 5(2):136-143, 2008.

[24] A. Richer and A. Adler. Eddy current based flexible sensor for contactless measurement of breathing. Instrumentation and Measurement Technology Conference, 2005. IMTC 2005. Proceedings of the IEEE, 1:257-260, May 2005.

[25] Steven D. Roach. Designing and building an eddy current position sensor. Sensors, September 1998.

[26] Javier Rosell, Roberto Casanas, and Hermann Scharfetter. Sensitivity maps and system requirements for magnetic induction tomography using a planar gradiometer. Physiological Measurement, 22(1):121-130, 2001.

[27] Adel S. Sedra and Kenneth C. Smith. Microelectronic Circuits. Oxford University Press, New York, 5th edition, 2003. 
[28] J Siivola. New noninvasive piezoelectric transducer for recording of respiration, heart rate and body movements. Med. Biol. Eng. Comput., 27(4):423-424, July 1989.

[29] M. Steffen, A. Aleksandrowicz, and S. Leonhardt. Mobile noncontact monitoring of heart and lung activity. Biomedical Circuits and Systems, IEEE Transactions on, 1(4):250-257, Dec. 2007.

[30] Alfred Steinschneider. Prolonged apnea and the sudden infant death syndrome: Clinical and laboratory observations. Pediatrics, 50(4):646-654, 1972.

[31] Peter P. Tarjan and Richard McFee. Electrodeless measurements of the effective resistivity of the human torso and head by magnetic induction. Biomedical Engineering, IEEE Transactions on, BME-15(4):266-278, Oct. 1968.

[32] M. Uenoyama, T. Matsui, K. Yamada, S. Suzuki, B. Takase, S. Suzuki, M. Ishihara, and M. Kawakami. Non-contact respiratory monitoring system using a ceiling-attached microwave antenna. Med Biol Eng Comput, 44(9):835-840, September 2006.

[33] B. Ulker and N.G. Gencer. Implementation of a data acquisition system for contactless conductivity imaging. Engineering in Medicine and Biology Magazine, IEEE, 21(5):152-155, Sep/Oct 2002.

[34] S Watson, A Morris, R J Williams, H Griffiths, and W Gough. A primary field compensation scheme for planar array magnetic induction tomography. Physiological Measurement, 25(1):271-279, 2004.

[35] H A Wheeler. Simple inductance formulas for radio coils. Proc Inst Radio Eng, 16(10):1398-1400, October 1928. 